\title{
Search for new physics in final states with a lepton and missing transverse energy in $p p$ collisions at the $\mathrm{LHC}$
}

\author{
S. Chatrchyan et al.* \\ (CMS Collaboration) \\ (Received 12 February 2013; published 8 April 2013)
}

\begin{abstract}
This Letter describes the search for an enhanced production rate of events with a charged lepton and a neutrino in high-energy $p p$ collisions at the LHC. The analysis uses data collected with the CMS detector, with an integrated luminosity of $5.0 \mathrm{fb}^{-1}$ at $\sqrt{s}=7 \mathrm{TeV}$, and a further $3.7 \mathrm{fb}^{-1}$ at $\sqrt{s}=8 \mathrm{TeV}$. No evidence is found for an excess. The results are interpreted in terms of limits on a heavy charged gauge boson $\left(W^{\prime}\right)$ in the sequential standard model, a split universal extra dimension model, and contact interactions in the helicity-nonconserving model. For the last, values of the binding energy below 10.5 (8.8) $\mathrm{TeV}$ in the electron (muon) channel are excluded at a 95\% confidence level. Interpreting the $\ell \nu$ final state in terms of a heavy $W^{\prime}$ with standard model couplings, masses below $2.90 \mathrm{TeV}$ are excluded.
\end{abstract}

DOI: 10.1103/PhysRevD.87.072005

PACS numbers: $12.60 . \mathrm{Cn}, 13.85 . \mathrm{Rm}, 14.70 . \mathrm{Pw}$

\section{INTRODUCTION}

New heavy gauge bosons are predicted by various extensions of the standard model (SM). The sequential standard model (SSM) [1] postulates the existence of a $W^{\prime}$ boson, a heavy analogue of the $W$. In such a theory, the $W^{\prime}$ is expected to appear as a narrow resonance with decay modes and branching fractions similar to those of the $W$. For $W^{\prime}$ masses above $180 \mathrm{GeV}$, where the $t \bar{b}$ decay channel opens up, the predicted branching fraction is about $8.5 \%$ for each of the leptonic final states. Previous searches [2,3] with $p p$ collision data at $\sqrt{s}=7 \mathrm{TeV}$ by the Compact Muon Solenoid (CMS) and ATLAS experiments at the Large Hadron Collider (LHC), based on an integrated luminosity of up to $5 \mathrm{fb}^{-1}$, have excluded SSM $W^{\prime}$ bosons with masses up to $2.6 \mathrm{TeV}$.

Other models for new physics predict the same final state, such as those with universal extra dimensions (UED) and bulk fermions, or split UED [4,5]. Such models of extended space-time assume one additional compact dimension of radius $R$. The split-UED parameter space is defined by $1 / R$ and $\mu$, with $\mu$ being the bulk mass parameter of the fermion field in five dimensions. For suitable nonzero values of $\mu$, as assumed by splitUED models, the cross sections are sufficiently large to allow observation by LHC experiments. All SM particles have corresponding Kaluza-Klein (KK) partners, for instance $W_{\mathrm{KK}}^{n}$, where $n$ denotes the $n$th KK excitation mode. Only KK-even modes of $W_{\mathrm{KK}}^{n}$ couple to SM fermions, owing to KK-parity conservation [6]. Modes with $n \geq 4$ have a smaller cross section and are not expected to be

*Full author list given at the end of the article.

Published by the American Physical Society under the terms of the Creative Commons Attribution 3.0 License. Further distribution of this work must maintain attribution to the author(s) and the published article's title, journal citation, and DOI. accessible at $\sqrt{s}=8 \mathrm{TeV}$, hence the only mode considered is $n=2$.

Motivated by the observation of mass hierarchies in the fermion sector, theories have been developed where leptons and quarks are composite objects [7]. At energies much lower than the binding energy of these fundamental constituents, typically called $\Lambda$, quark and lepton compositeness would manifest itself as a four-fermion contact interaction (CI). One of the possible contact interactions between two quarks, a neutrino, and a charged lepton, is described by the helicity-nonconserving (HNC) model [8]. The corresponding cross section is proportional to the square of the partonic center-of-mass energy and to $\Lambda^{-4}$. While CDF has set a limit on $\Lambda>2.81 \mathrm{TeV}$ [9] based on a final state with an electron and a neutrino, no limit in the HNC model has yet been set in the muon channel.

In this Letter, a search is presented for an excess of events with an isolated charged lepton (an electron or muon) and a neutrino in the final state, using the CMS detector. The data sample corresponds to an integrated luminosity of $5.0 \mathrm{fb}^{-1}$ at $\sqrt{s}=7 \mathrm{TeV}$ collected in 2011 , and $3.7 \mathrm{fb}^{-1}$ at $\sqrt{s}=8 \mathrm{TeV}$ collected in 2012. The CMS 2011 result has been published in Ref. [2].

\section{THE CMS DETECTOR}

The central feature of the CMS apparatus is a superconducting solenoid of $6 \mathrm{~m}$ internal diameter, providing a magnetic field of $3.8 \mathrm{~T}$. Within the superconducting solenoid volume are a silicon pixel and strip tracker, a lead tungstate crystal electromagnetic calorimeter, and a brass and scintillator hadron calorimeter. Muons are measured in gas-ionization detectors embedded in the steel return yoke. Extensive forward calorimetry complements the coverage provided by the barrel and end cap detectors. A more detailed description of the CMS detector can be found in Ref. [10]. CMS uses a right-handed coordinate system, with the origin at the nominal interaction point, the $x$ 
axis pointing to the center of the LHC, the $y$ axis pointing up (perpendicular to the LHC plane), and the $z$ axis along the anticlockwise-beam direction. The polar angle $\theta$ is measured from the positive $z$ axis and the azimuthal angle $\phi$ is measured in the $x-y$ plane.

\section{THE SEARCH STRATEGY}

Candidate events with at least one high transverse momentum $\left(p_{\mathrm{T}}\right)$ electron or muon are selected using singlelepton triggers. Isolated high- $p_{\mathrm{T}}$ leptons are reconstructed
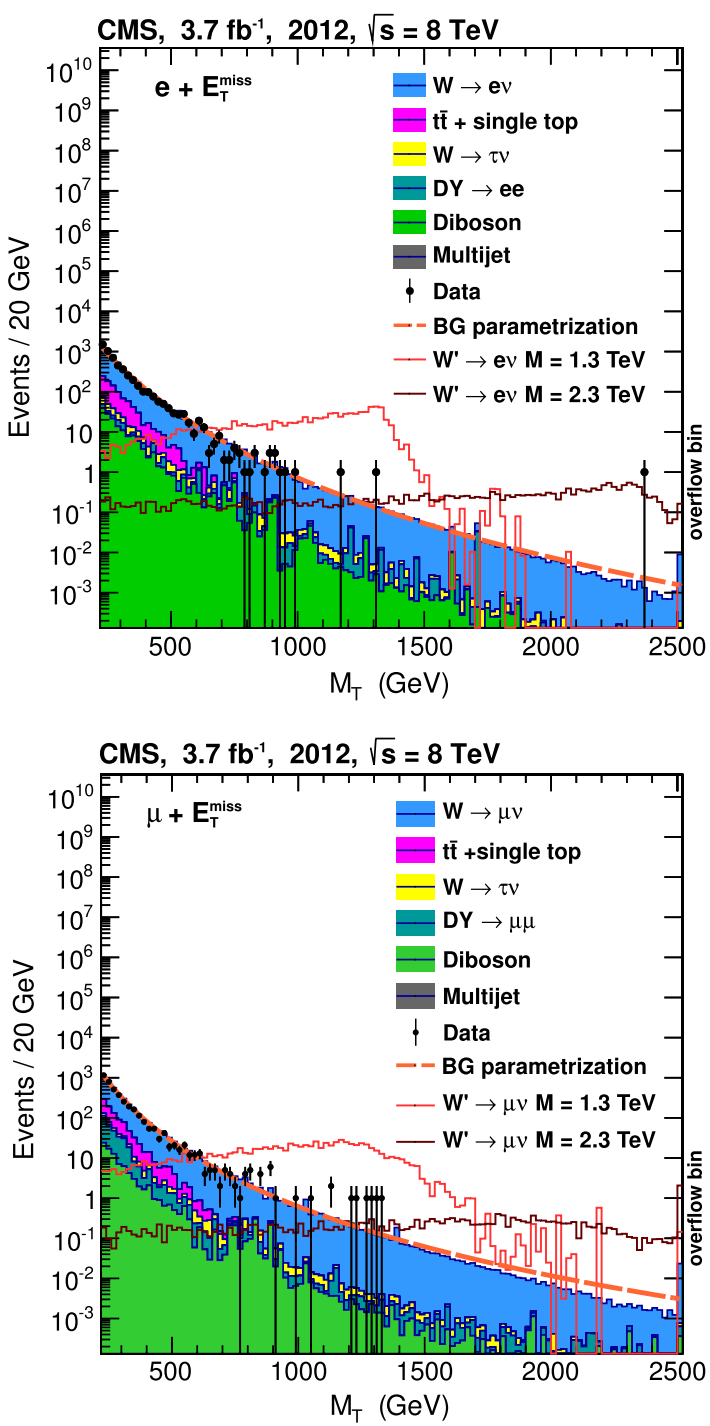

FIG. 1 (color online). Observed lepton- $E_{\mathrm{T}}^{\text {miss }}$ transverse mass distributions in the electron (top) and the muon (bottom) channel. The dashed lines show the parametrization of the background as described in the text (labeled as BG parametrization in the legend). Simulated signal distributions for a SSM $W^{\prime}$ are also shown, including detector resolution effects. The simulated background labeled as "diboson" includes $W W, Z Z$, and $W Z$ contributions. The last bin contains the contributions of all bins above the displayed range. using very stringent quality criteria while the neutrino gives rise to experimentally observed missing transverse energy $\left(E_{\mathrm{T}}^{\text {miss }}\right)$. The details on lepton identification and $E_{\mathrm{T}}^{\text {miss }}$ reconstruction, as optimized for the 2011 analysis, can be found in Ref. [2]. The discriminating variable of this analysis is the transverse mass $M_{\mathrm{T}}$ of the lepton- $E_{\mathrm{T}}^{\text {miss }}$ system, calculated as

$$
M_{\mathrm{T}}=\sqrt{2 \cdot p_{\mathrm{T}}^{\ell} \cdot E_{\mathrm{T}}^{\mathrm{miss}} \cdot\left(1-\cos \Delta \phi_{\ell, \nu}\right)},
$$

where $\Delta \phi_{\ell, \nu}$ is the azimuthal angle between the charged lepton's transverse momentum $p_{\mathrm{T}}^{\ell}$ and the $E_{\mathrm{T}}^{\text {miss }}$ direction.

In $W^{\prime}$ decays, as well as for the other models considered, the lepton and $E_{\mathrm{T}}^{\text {miss }}$ are expected to be almost back to back in the transverse plane, and balanced in transverse energy. Additional kinematic criteria therefore select events with a ratio of the lepton $p_{\mathrm{T}}^{\ell}$ and the $E_{\mathrm{T}}^{\text {miss }}, 0.4<p_{\mathrm{T}}^{\ell} / E_{\mathrm{T}}^{\text {miss }}<1.5$, along with the requirement of the angular difference, $\left|\Delta \phi_{\ell, \nu}-\pi\right|<0.2 \pi$. For simulated SSM $W^{\prime}$ events with masses between 0.5 and $2.5 \mathrm{TeV}$ passing these selection criteria, the signal efficiency (including 90\% geometrical acceptance) is found to be $70 \%-75 \%$ with $2 \%$ uncertainty in the electron channel and $67 \%-72 \%$ with $1 \%$ uncertainty in the muon channel. For the HNC contact-interaction model the signal efficiency is independent of the interaction scale $\Lambda$ and has been determined from simulation to be $80 \%$ with $1 \%$ uncertainty for the electron channel and $77 \%$ with $4 \%$ uncertainty for the muon channel. The transverse mass distributions for accepted SM events in the electron and muon channels are shown in Fig. 1, along with two example $W^{\prime}$ signals. The observed event with the highest transverse mass in the electron channel has $M_{\mathrm{T}}=2.38 \pm$ $0.05 \mathrm{TeV}$ based on $E_{\mathrm{T}}=1.2 \mathrm{TeV}$ with $1 \%$ uncertainty. In the muon channel the maximum transverse mass is $M_{\mathrm{T}}=$ $1.33 \pm 0.03 \mathrm{TeV}$ with a measured $p_{\mathrm{T}}^{\ell}$ of $690 \pm 22 \mathrm{GeV}$. The two types of processes, $W^{\prime}$ production and compositeness, can be distinguished by examining the shape of the lepton- $E_{\mathrm{T}}^{\text {miss }}$ transverse mass spectrum. Both processes manifest themselves through an excess of events at the

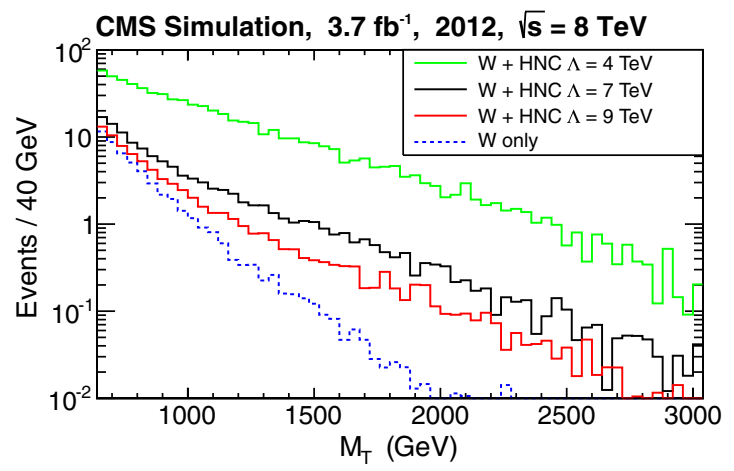

FIG. 2 (color online). Simulated transverse mass distribution shown for contact interaction (HNC model), in the muon $+E_{\mathrm{T}}^{\text {miss }}$ channel at generator level for CI signals with $\Lambda=4,7$, and $9 \mathrm{TeV}$, and the SM background $(W \rightarrow \mu \nu)$. 
high end of the spectrum. The $W^{\prime}$ signal events are expected to concentrate in a Jacobian peak around the $W^{\prime}$ mass, as shown in Fig. 1. Compositeness would rather yield an unstructured excess, with the excess relative to the standard model contribution increasing as a function of $M_{\mathrm{T}}$, as shown in Fig. 2.

\section{SIGNAL AND BACKGROUNDS}

The $W^{\prime}$ (this also includes $W_{\mathrm{KK}}^{n=2}$ ) and CI signals are generated at leading order (LO) with PYTHIA 6.4.26 [11] using the CTEQ6L1 [12] parton distribution functions. They are scaled to the next-to-next-to-leading order (NNLO) cross section calculated with FEWZ $[13,14]$ for each $W^{\prime}\left(W_{\mathrm{KK}}^{n=2}\right)$ mass point. In the absence of higher-order calculations, LO cross sections are used for the CI model.

The primary source of background is the off-peak, high- $M_{\mathrm{T}}$ tail of the standard model $W \rightarrow \ell \nu$ decays. Other important backgrounds arise from $t \bar{t}$, Drell-Yan, and diboson $(W W, W Z, Z Z)$ events. An important background also comes from QCD multijet processes, but this is largely removed by the analysis selections and therefore cannot be readily distinguished in Fig. 1. Contributions from decays with $\tau$ leptons in the final state that subsequently decay to an electron or muon and neutrinos are considered as well, but found to be negligible. The PYTHIA 6 generator is used for all background processes except for $t \bar{t}$, which is generated with MADGRAPH 4 [15] in combination with PYTHIA. The numbers of Monte Carlo events are normalized using the integrated luminosity of the recorded data and with NNLO cross sections, except diboson and multijet samples, for which the next-to-leading order and LO cross sections are used, respectively. In all event samples, additional minimum bias interactions are superimposed onto the main background processes to match the luminosity profile of the analyzed data set.



FIG. 3 (color online). Limits on the cross section times the single channel branching fraction $(\sigma \times \mathcal{B})$ for heavy bosons based on the 2012 data for the electron and the muon channels. For the individual channels, only the observed limits are shown. For the combination, the observed limit, the expected limit, the $1 \sigma$, and the $2 \sigma$ bands are displayed. The model assumes equal branching fractions for the electron and the muon channel, hence the combination corresponds to doubling the number of events. All limits are displayed for the single channel branching fraction. The $W^{\prime}$ mass limits are derived with a Bayesian method for the models of a SSM $W^{\prime}$ and $W_{\mathrm{KK}}^{n=2}$ in split UED. The $W_{\mathrm{KK}}^{n=2}$ is the lowest mass state that can couple to SM fermions and has the same final state as the SM-like $W^{\prime}$. Because it has even $\mathrm{KK}$ parity, it can be produced singly.

The background prediction is based on the transverse mass distributions of these simulations, as seen in Fig. 1. The summed background distribution is parametrized with a function of form $f\left(M_{\mathrm{T}}\right)=\frac{a}{\left(M_{\mathrm{T}}+b\right)^{c}}$ to avoid event

TABLE I. Data, background, and signal event yields for different transverse mass thresholds.

\begin{tabular}{|c|c|c|c|}
\hline & $M_{\mathrm{T}}>1.0 \mathrm{TeV}$ & $M_{\mathrm{T}}>1.5 \mathrm{TeV}$ & $M_{\mathrm{T}}>2.0 \mathrm{TeV}$ \\
\hline \multicolumn{4}{|c|}{ Electron channel } \\
\hline Data & 3 & 1 & 1 \\
\hline SM background & $6.8 \pm 0.4$ & $0.69_{-0.04}^{+0.05}$ & $0.13 \pm 0.02$ \\
\hline$M_{W^{\prime}}=2.5 \mathrm{TeV}$ & $9.7 \pm 0.3$ & $7.5 \pm 0.2$ & $4.7 \pm 0.2$ \\
\hline$M_{W^{\prime}}=3 \mathrm{TeV}$ & $1.98 \pm 0.06$ & $1.5 \pm 0.05$ & $1.15_{-0.04}^{+0.05}$ \\
\hline $\mathrm{CI}, \Lambda=4 \mathrm{TeV}$ & $220 \pm 5$ & $72 \pm 3$ & $21 \pm 1$ \\
\hline CI, $\Lambda=9 \mathrm{TeV}$ & $8.6 \pm 0.2$ & $2.8 \pm 0.1$ & $0.81_{-0.04}^{+0.05}$ \\
\hline \multicolumn{4}{|c|}{ Muon channel } \\
\hline Data & 9 & 0 & 0 \\
\hline SM background & $6.5_{-1.5}^{+1.9}$ & $0.92_{-0.32}^{+0.49}$ & $0.20_{-0.08}^{+0.14}$ \\
\hline$M_{W^{\prime}}=2.5 \mathrm{TeV}$ & $9.4 \pm 0.3$ & $7.0 \pm 0.5$ & $4.1_{-0.7}^{+0.8}$ \\
\hline$M_{W^{\prime}}=3 \mathrm{TeV}$ & $1.9 \pm 0.1$ & $1.4 \pm 0.1$ & $0.94_{-0.10}^{+0.12}$ \\
\hline $\mathrm{CI}, \Lambda=4 \mathrm{TeV}$ & $240 \pm 20$ & $80_{-13}^{+15}$ & $28_{-6}^{+8}$ \\
\hline $\mathrm{CI}, \Lambda=9 \mathrm{TeV}$ & $34 \pm 3$ & $12_{-2}^{+3}$ & $3.5_{-0.9}^{+1.2}$ \\
\hline
\end{tabular}


TABLE II. Exclusion limits in TeV on the SSM $W^{\prime}$ mass for the electron and muon channel as well as their combinations based on $5.0 \mathrm{fb}^{-1}$ of 2011 data at $\sqrt{s}=7 \mathrm{TeV}$ and $3.7 \mathrm{fb}^{-1}$ of 2012 data at $\sqrt{s}=8 \mathrm{TeV}$.

\begin{tabular}{lcccccc}
\hline \hline & Obs. & Exp. & Obs. & Exp. & Obs. & Exp. \\
Channel & 2011 & 2011 & 2012 & 2012 & $2011+2012$ & $2011+2012$ \\
\hline$e$ & 2.40 & 2.45 & 2.60 & 2.70 & 2.70 & 2.75 \\
$\mu$ & 2.40 & 2.45 & 2.75 & 2.65 & 2.75 & 2.70 \\
$e+\mu$ & 2.50 & 2.60 & 2.85 & 2.80 & 2.90 & 2.90 \\
\hline \hline
\end{tabular}

fluctuations due to the limited size of the Monte Carlo event sample, also shown in Fig. 1. In the low mass control region, e.g. for $M_{\mathrm{T}}>500 \mathrm{GeV}$, the background prediction in the electron channel is 169 events from a direct count and $155 \pm 16$ events from the parametrization. The corresponding event numbers in the muon channel are 162 and $138 \pm 12$, from the direct count and the parametrization, respectively. The number of observed events in each channel is 153. The expected background due to SM processes drops significantly with increasing $M_{\mathrm{T}}$. The number of events observed and the number expected from the parametrization, above three characteristic high $M_{\mathrm{T}}$ thresholds, are shown in Table I for the electron and the muon channels. Also summarized are the expected numbers of $W^{\prime}$ and CI signal events, as evaluated from simulation, including systematic uncertainties due to imperfections in the description of the detector performance.

\section{RESULTS AND EXCLUSION LIMITS}

For determining the exclusion limits, systematic uncertainties on the signal and background yields are included via nuisance parameters with a log-normal prior distribution in the same way as in our previous analysis from Ref. [2]. The lepton-energy calibration and resolution distort the transverse mass spectrum. Uncertainties in the energy scale and the energy resolution make comparable contributions to the overall uncertainty in the electron channel: the energy scale has an uncertainty of $1(3) \%$ in the barrel (end caps) and the resolution has an uncertainty of 1.4(3)\% for the barrel (end caps) [16]. For muons, the dominant uncertainty stems from the momentum scale, which is taken to be $5 \% \times p_{\mathrm{T}} / \mathrm{TeV}$ [17] and results in larger errors on the background and signal event yields in the muon channel, as can be seen in Table I. The muon $p_{\mathrm{T}}$ resolution has been determined with cosmic ray muons to be $10 \%$ at high $p_{\mathrm{T}}$ with an uncertainty of $0.6 \%$ [17].

Similarly, the impact of the $E_{\mathrm{T}}^{\text {miss }}$ energy scale is modeled by shifting the hadronic component event by event by $10 \%$, while the resolution is taken into account by a $10 \%$ smearing. In all cases, the impact on the expected number of signal events is around $1 \%$ for each source of uncertainty. The background parametrization procedure is repeated using the distorted distributions. Additionally, an uncertainty is derived by fitting the undistorted background with two different functions. The estimates shown in Table I include a systematic uncertainty that covers the range of results from these fits, with the statistical uncertainty coming from the fit to the original distribution. Additionally, an uncertainty of $4.4 \%$ is considered on the integrated luminosity [18].

The number of data events above a transverse mass threshold $M_{\mathrm{T}}^{\min }$ is compared to the expected number of signal and background events, with the $M_{\mathrm{T}}^{\text {min }}$ threshold being optimized for the best expected exclusion limit, using steps of $50 \mathrm{GeV}$. Very similar results are achieved
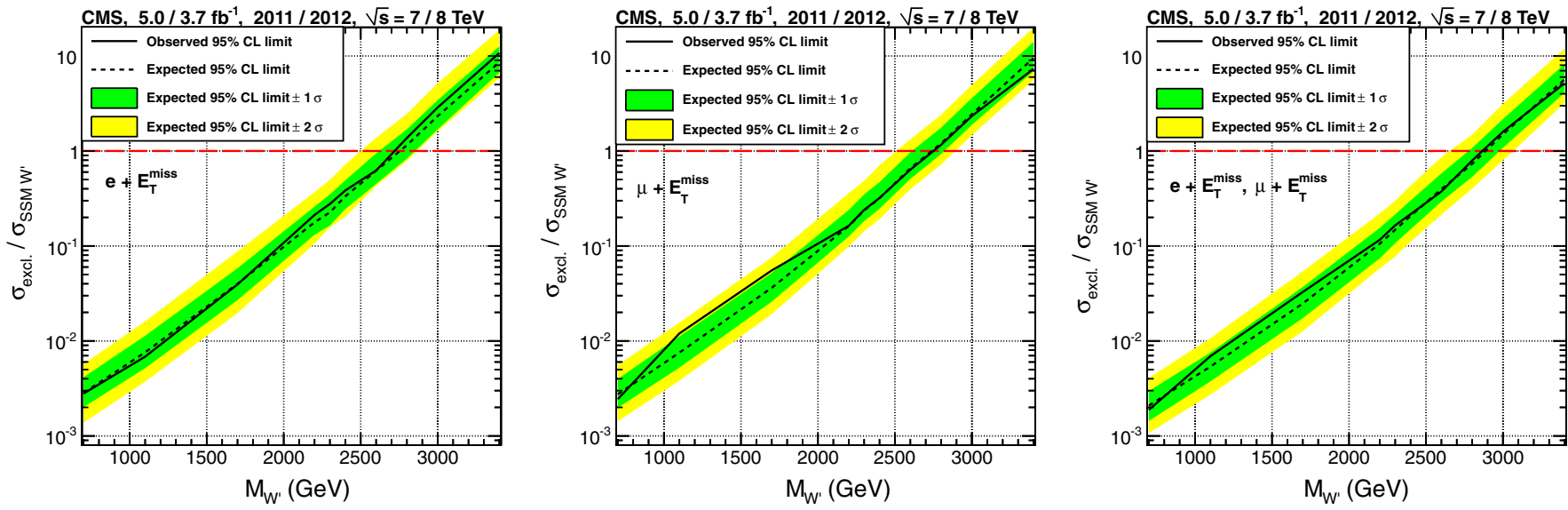

FIG. 4 (color online). Combination of 2011 and 2012 data for the electron and the muon channels using $5.0 \mathrm{fb}^{-1}$ of data at $\sqrt{\mathrm{s}}=$ $7 \mathrm{TeV}$ from 2011 and $3.7 \mathrm{fb}^{-1}$ of data at $\sqrt{s}=8 \mathrm{TeV}$ from 2012. The left and middle plots show the individual combinations for the electron (left) and muon channel (right). To the right the combination of both is displayed. The limits are derived with a Bayesian method. Plotted is the signal strength modifier $\sigma_{\text {excl }} / \sigma_{\mathrm{SSM} W^{\prime}}$ as a function of the $W^{\prime}$ mass, where $\sigma_{\text {excl }}$ is the cross section excluded at a $95 \%$ C.L. and $\sigma_{\mathrm{SSM} W^{\prime}}$ is the cross section predicted by the SSM. All $W^{\prime}$ mass points below the ratio $\sigma_{\text {excl }} / \sigma_{\mathrm{SSM} W^{\prime}}=1$, shown as a red dashed line, are excluded in the sequential standard model. 




FIG. 5 (color online). The 95\% C.L. limits on the split-UED parameters $\mu$ and $1 / R$ derived from the $W^{\prime}$ mass limits taking into account the corresponding width of the $W_{\mathrm{KK}}^{n=2}$. For the $3.7 \mathrm{fb}^{-1}$ of 2012 data, the individual limits for the electron and muon channels are shown together with their combination, improving the excluded parameter space based on the 2011 data shown in yellow.

when optimizing for discovery. The same optimization procedure is applied for the $W^{\prime}$ and CI searches but the optimized $M_{\mathrm{T}}$ thresholds are specific to each analysis. For a $W^{\prime}$ mass of up to $2.5 \mathrm{TeV}$ the optimization tends to select a value for $M_{\mathrm{T}}^{\min }$ a little below the peak of the Jacobian, retaining most of the events in the peak. To keep a significant signal contribution for higher masses, the $M_{\mathrm{T}}^{\text {min }}$ threshold is shifted to lower values owing to the increasing off-shell fraction. For CI no such effect exists and the optimized $M_{\mathrm{T}}^{\min }$ threshold is roughly constant around $1 \mathrm{TeV}$.

No significant excess has been observed in the data, and upper limits are set on the production cross section times the branching fraction $\sigma \times \mathcal{B}\left(W^{\prime} \rightarrow \ell \nu\right)$, with $\ell=e$ or $\mu$, using a Bayesian method with the assumption of a flat prior for the parameter of interest. The expected and observed upper cross section limits on a SSM $W^{\prime}$, at $95 \%$ confidence level (C.L.), for $\sqrt{s}=8 \mathrm{TeV}$ data, are shown in Fig. 3 for both channels and their combination. Using the central value of the theoretical NNLO SSM $W^{\prime}$ cross section times branching fraction, which is assumed to be identical in the two channels, we exclude masses less than $2.60 \mathrm{TeV}$ in the electron and $2.75 \mathrm{TeV}$ in the muon channel. The expected limits are $2.70 \mathrm{TeV}$ and $2.65 \mathrm{TeV}$, respectively. The observed limit for $W^{\prime} \rightarrow e \nu$ is slightly lower than the expectation because of one event with a transverse mass of $2.3 \mathrm{TeV}$ (Fig. 1). Combining both channels, the limit increases to $2.85 \mathrm{TeV}$ (Table II). To further enhance the sensitivity, the new results can be combined with the published [2] $\sqrt{s}=7 \mathrm{TeV}$ results, based on an integrated
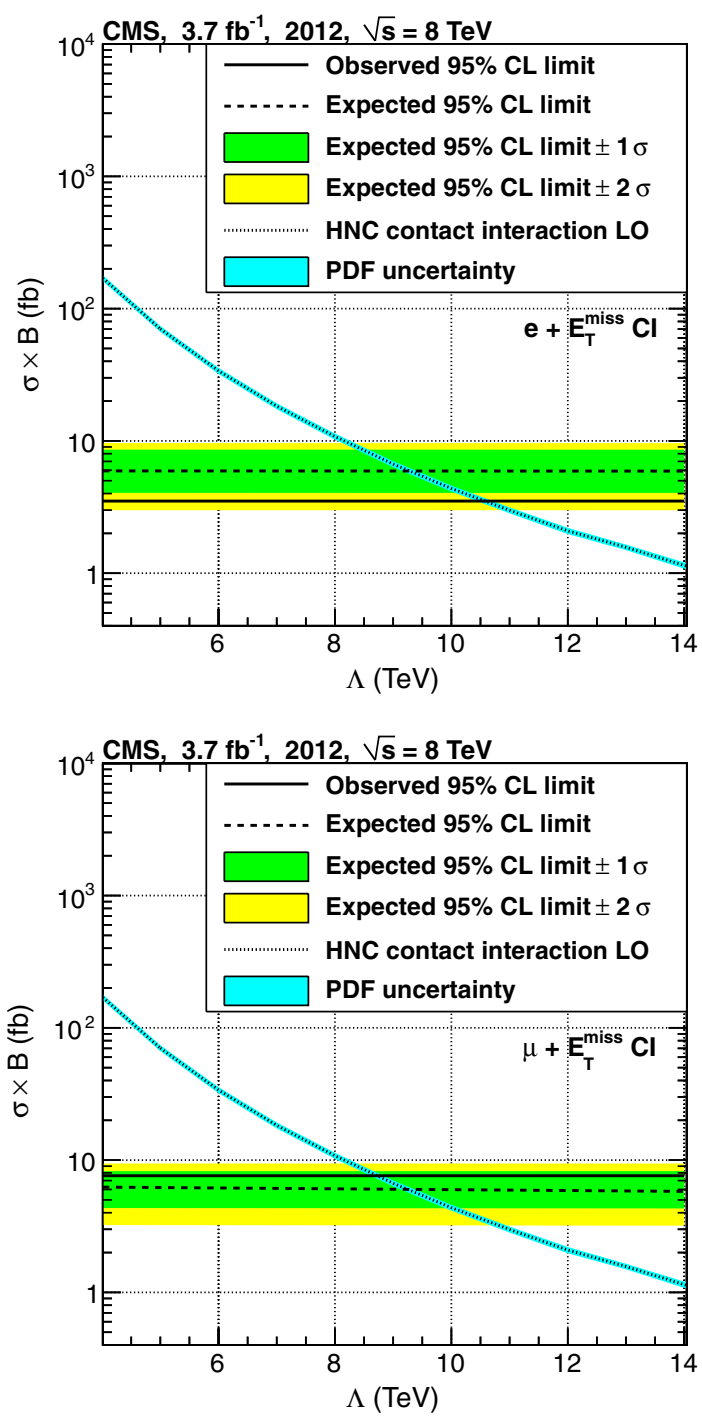

FIG. 6 (color online). Bayesian limits on the cross section times the single channel branching fraction $(\sigma \times \mathcal{B})$ for contact interactions (HNC model) in the electron $+E_{\mathrm{T}}^{\text {miss }}$ (top) and the muon $+E_{\mathrm{T}}^{\text {miss }}$ channel (bottom) based on an integrated luminosity of $3.7 \mathrm{fb}^{-1}$ of $\sqrt{s}=8 \mathrm{TeV}$ data. The expected limit in either channel excludes values of the new interaction scale $\Lambda<9 \mathrm{TeV}$. The observed limit, driven by the data, excludes $\Lambda<10.5 \mathrm{TeV}$ (8.8) in the electron (muon) channel. The signal efficiency is independent of $\Lambda$.

luminosity of $5 \mathrm{fb}^{-1}$, thus extending the limit to $2.90 \mathrm{TeV}$ as shown in Fig. 4 and Table II.

The limit can be reinterpreted in terms of the $W_{\mathrm{KK}}^{n=2}$ mass, as shown in Fig. 3, for values of the Dirac mass term $\mu=0.05 \mathrm{TeV}$ and $\mu=10 \mathrm{TeV}$ and directly translated to bounds on the split-UED parameter space, $(1 / R, \mu)$ (Fig. 5). The four-fermion contact interaction of the HNC model is excluded for values of $\Lambda<10.5 \mathrm{TeV}$ in the electron channel and $\Lambda<8.8 \mathrm{TeV}$ in the muon channel (Fig. 6). While the expected sensitivity is comparable in both search channels, the exclusion limit in the electron 
TABLE III. Exclusion limits for other model interpretations for the electron and muon channel or their combination based on $3.7 \mathrm{fb}^{-1}$ of 2012 data at $\sqrt{s}=8 \mathrm{TeV}$.

\begin{tabular}{lccc}
\hline \hline Model & Channel & Observed limit & Expected limit \\
\hline$W_{\mathrm{KK}}^{n=2} \mu=0.05 \mathrm{TeV}$ & $e+\mu$ & $\mathrm{m}_{\mathrm{W}_{\mathrm{KK}}^{\mathrm{n}=2}}<1.5 \mathrm{TeV}$ & $\mathrm{m}_{\mathrm{W}_{\mathrm{KK}}^{\mathrm{n}=2}}<1.4 \mathrm{TeV}$ \\
$\mathrm{W}_{\mathrm{KK}}^{n=2} \mu=10.0 \mathrm{TeV}$ & $\mathrm{e}+\mu$ & $\mathrm{m}_{\mathrm{W}_{\mathrm{KK}}^{\mathrm{n}=2}}<3.3 \mathrm{TeV}$ & $\mathrm{m}_{\mathrm{W}_{\mathrm{KK}}^{\mathrm{n}=2}}<3.2 \mathrm{TeV}$ \\
$\mathrm{HNC} \mathrm{CI}$ & $e$ & $\Lambda<0.5 \mathrm{TeV}$ & $\Lambda<9.0 \mathrm{TeV}$ \\
$\mathrm{HNC}$ CI & $\mu$ & $\Lambda<8.8 \mathrm{TeV}$ & $\Lambda<9.0 \mathrm{TeV}$ \\
\hline \hline
\end{tabular}

channel is higher because of a downward fluctuation of data (see Table I) in the search region of $M_{\mathrm{T}}>1 \mathrm{TeV}$. These limits are summarized in Table III.

\section{SUMMARY}

A search for new physics has been carried out using $p p$ collisions recorded with the CMS detector at center-ofmass energies of $\sqrt{s}=7$ and $8 \mathrm{TeV}$. The transverse mass spectrum of either electrons or muons and missing transverse energy has been measured. The observed spectra are consistent with the standard model expectation, and mass limits on sequential standard model $W^{\prime}$ and $W_{\mathrm{KK}}^{n=2}$ have been set at the $95 \%$ C.L. These are the most stringent limits to date. For the first time, a limit on the compositeness scale $\Lambda$ has been set for contact interactions in the HNC model based on the final state with a muon and $E_{\mathrm{T}}^{\text {miss }}$. The corresponding exclusion limit in the electron channel significantly improves the existing one.

\section{ACKNOWLEDGMENTS}

We congratulate our colleagues in the CERN accelerator departments for the excellent performance of the LHC and thank the technical and administrative staffs at CERN and at other CMS institutes for their contributions to the success of the CMS effort. In addition, we gratefully acknowledge the computing centers and personnel of the Worldwide LHC Computing Grid for delivering so effectively the computing infrastructure essential to our analyses. Finally, we acknowledge the enduring support for the construction and operation of the LHC and the CMS detector provided by the following funding agencies: BMWF and FWF (Austria); FNRS and FWO (Belgium); CNPq, CAPES, FAPERJ, and FAPESP (Brazil); MEYS (Bulgaria); CERN; CAS, MoST, and NSFC (China); COLCIENCIAS (Colombia); MSES (Croatia); RPF (Cyprus); MoER, SF0690030s09, and ERDF (Estonia); Academy of Finland, MEC, and HIP (Finland); CEA and CNRS/IN2P3 (France); BMBF, DFG, and HGF (Germany); GSRT (Greece); OTKA and NKTH (Hungary); DAE and DST (India); IPM (Iran); SFI (Ireland); INFN (Italy); NRF and WCU (Republic of Korea); LAS (Lithuania); CINVESTAV, CONACYT, SEP, and UASLP-FAI (Mexico); MSI (New Zealand); PAEC (Pakistan); MSHE and NSC (Poland); FCT (Portugal); JINR (Armenia, Belarus, Georgia, Ukraine, Uzbekistan); MON, RosAtom, RAS, and RFBR (Russia); MSTD (Serbia); SEIDI and CPAN (Spain); Swiss Funding Agencies (Switzerland); NSC (Taipei); ThEPCenter, IPST and NSTDA (Thailand); TUBITAK and TAEK (Turkey); NASU (Ukraine); STFC (United Kingdom); DOE and NSF (USA).
[1] G. Altarelli, B. Mele, and M. Ruiz-Altaba, Z. Phys. C 45, 109 (1989).

[2] CMS Collaboration, J. High Energy Phys. 08 (2012) 023.

[3] ATLAS Collaboration, Eur. Phys. J. C 72, 2241 (2012).

[4] C.-R. Chen, M. M. Nojiri, S.C. Park, J. Shu, and M. Takeuchi, J. High Energy Phys. 09 (2009), 078.

[5] K. Kong, S. C. Park, and T. G. Rizzo, J. High Energy Phys. 04 (2010), 081.

[6] T. Appelquist, H.-C. Cheng, and B. A. Dobrescu, Phys. Rev. D 64, 035002 (2001).

[7] H. Terazawa, M. Yasue, K. Akama, and M. Hayashi, Phys. Lett. 112B, 387 (1982).

[8] K. D. Lane, F. E. Paige, T. Skwarnicki, and W. J. Womersley, Phys. Rep. 278, 291 (1997).
[9] T. Aolder et al. (CDF Collaboration), Phys. Rev. Lett. 87, 231803 (2001).

[10] CMS Collaboration, JINST 3, S08004 (2008).

[11] T. Sjostrand, S. Mrenna, and P.Z. Skands, J. High Energy Phys. 05 (2006), 026.

[12] J. Pumplin, D. R. Stump, J. Huston, H. L. Lai, P. Nadolsky, and W. K. Tung, J. High Energy Phys. 07 (2002) 012.

[13] R. Gavin, Y. Li, F. Petriello, and S. Quackenbush, Comput. Phys. Commun. 182, 2388 (2011).

[14] S. Quackenbush, R. Gavin, Y. Li, and F. Petriello, Comput. Phys. Commun. 184, 209 (2013).

[15] J. Alwall, P. Demin, S. de Visscher, R. Frederix, M. Herquet, F. Maltoni, T. Plehn, D. L. Rainwater, and T. Stelzer, J. High Energy Phys. 09 (2007) 028. 
[16] CMS Collaboration, CMS Detector Performance Summary, Report No. CMS-DP-2012-007, 2012, http:// cds.cern.ch/record/1457922.

[17] CMS Collaboration JINST 7, P10002 (2012).
[18] CMS Collaboration, CMS Physics Analysis Summary, Report No. CMS-PAS-LUM-12-001， 2012， http:// cds.cern.ch/record/1457922.

S. Chatrchyan, ${ }^{1}$ V. Khachatryan, ${ }^{1}$ A. M. Sirunyan, ${ }^{1}$ A. Tumasyan, ${ }^{1}$ W. Adam,${ }^{2}$ E. Aguilo, ${ }^{2}$ T. Bergauer, ${ }^{2}$ M. Dragicevic, ${ }^{2}$ J. Erö, ${ }^{2}$ C. Fabjan, ${ }^{2, b}$ M. Friedl, ${ }^{2}$ R. Frühwirth, ${ }^{2, b}$ V. M. Ghete, ${ }^{2}$ N. Hörmann, ${ }^{2}$ J. Hrubec, ${ }^{2}$ M. Jeitler, ${ }^{2, b}$ W. Kiesenhofer, ${ }^{2}$ V. Knünz, ${ }^{2}$ M. Krammer, ${ }^{2, b}$ I. Krätschmer, ${ }^{2}$ D. Liko, ${ }^{2}$ I. Mikulec, ${ }^{2}$ M. Pernicka, ${ }^{2, a}$ D. Rabady, ${ }^{2, \mathrm{c}}$ B. Rahbaran, ${ }^{2}$ C. Rohringer ${ }^{2}$ H. Rohringer, ${ }^{2}$ R. Schöfbeck, ${ }^{2}$ J. Strauss, ${ }^{2}$ A. Taurok, ${ }^{2}$ W. Waltenberger, ${ }^{2}$ C.-E. Wulz, ${ }^{2, b}$ V. Mossolov, ${ }^{3}$ N. Shumeiko, ${ }^{3}$ J. Suarez Gonzalez, ${ }^{3}$ S. Alderweireldt, ${ }^{4}$ M. Bansal, ${ }^{4}$ S. Bansal, ${ }^{4}$ T. Cornelis, ${ }^{4}$ E. A. De Wolf, ${ }^{4}$ X. Janssen,${ }^{4}$ S. Luyckx,${ }^{4}$ L. Mucibello, ${ }^{4}$ S. Ochesanu, ${ }^{4}$ B. Roland ${ }^{4}$ R. Rougny, ${ }^{4}$ M. Selvaggi, ${ }^{4}$ H. Van Haevermaet, ${ }^{4}$ P. Van Mechelen, ${ }^{4}$ N. Van Remortel, ${ }^{4}$ A. Van Spilbeeck, ${ }^{4}$ F. Blekman, ${ }^{5}$ S. Blyweert, ${ }^{5}$ J. D'Hondt, ${ }^{5}$ R. Gonzalez Suarez, ${ }^{5}$ A. Kalogeropoulos, ${ }^{5}$ M. Maes, ${ }^{5}$ A. Olbrechts, ${ }^{5}$ S. Tavernier, ${ }^{5}$ W. Van Doninck, ${ }^{5}$ P. Van Mulders, ${ }^{5}$ G. P. Van Onsem, ${ }^{5}$ I. Villella, ${ }^{5}$ B. Clerbaux,${ }^{6}$ G. De Lentdecker, ${ }^{6}$ V. Dero, ${ }^{6}$ A.P. R. Gay, ${ }_{6}^{6}$ T. Hreus, ${ }^{6}$ A. Léonard, ${ }^{6}$ P.E. Marage, ${ }^{6}$ A. Mohammadi, ${ }^{6}$ T. Reis, ${ }^{6}$ L. Thomas, ${ }^{6}$ C. Vander Velde, ${ }^{6}$ P. Vanlaer, ${ }^{6}$ J. Wang, ${ }^{6}$ V. Adler, ${ }^{7}$ K. Beernaert, ${ }^{7}$ A. Cimmino, ${ }^{7}$ S. Costantini, ${ }^{7}$ G. Garcia, ${ }^{7}$ M. Grunewald, ${ }^{7}$ B. Klein, ${ }^{7}$

J. Lellouch, ${ }^{7}$ A. Marinov, ${ }^{7}$ J. Mccartin, ${ }^{7}$ A. A. Ocampo Rios, ${ }^{7}$ D. Ryckbosch, ${ }^{7}$ M. Sigamani, ${ }^{7}$ N. Strobbe, ${ }^{7}$

F. Thyssen, ${ }^{7}$ M. Tytgat, ${ }^{7}$ S. Walsh, ${ }^{7}$ E. Yazgan, ${ }^{7}$ N. Zaganidis, ${ }^{7}$ S. Basegmez, ${ }^{8}$ G. Bruno, ${ }^{8}$ R. Castello, ${ }^{8}$ L. Ceard, ${ }^{8}$

C. Delaere, ${ }^{8}$ T. du Pree, ${ }^{8}$ D. Favart, ${ }^{8}$ L. Forthomme, ${ }^{8}$ A. Giammanco, ${ }^{8, d}$ J. Hollar, ${ }^{8}$ V. Lemaitre, ${ }^{8}$ J. Liao, ${ }^{8}$

O. Militaru, ${ }^{8}$ C. Nuttens, ${ }^{8}$ D. Pagano, ${ }^{8}$ A. Pin,${ }^{8}$ K. Piotrzkowski,${ }^{8}$ J. M. Vizan Garcia,${ }^{8}$ N. Beliy, ${ }^{9}$ T. Caebergs, ${ }^{9}$

E. Daubie, ${ }^{9}$ G. H. Hammad, ${ }^{9}$ G. A. Alves, ${ }^{10}$ M. Correa Martins Junior, ${ }^{10}$ T. Martins,${ }^{10}$ M. E. Pol, ${ }^{10}$ M. H. G. Souza, ${ }^{10}$

W. L. Aldá Júnior, ${ }^{11}$ W. Carvalho, ${ }^{11}$ J. Chinellato, ${ }^{11}$ A. Custódio, ${ }^{11}$ E. M. Da Costa, ${ }^{11}$ D. De Jesus Damiao, ${ }^{11}$

C. De Oliveira Martins, ${ }^{11}$ S. Fonseca De Souza, ${ }^{11}$ H. Malbouisson, ${ }^{11}$ M. Malek, ${ }^{11}$ D. Matos Figueiredo, ${ }^{11}$

L. Mundim, ${ }^{11}$ H. Nogima,${ }^{11}$ W. L. Prado Da Silva, ${ }^{11}$ A. Santoro, ${ }^{11}$ L. Soares Jorge, ${ }^{11}$ A. Sznajder,${ }^{11}$

E. J. Tonelli Manganote, ${ }^{11}$ A. Vilela Pereira,${ }^{11}$ T. S. Anjos, ${ }^{12 b}$ C. A. Bernardes,${ }^{12 b}$ F. A. Dias, ${ }^{12 a, e}$

T. R. Fernandez Perez Tomei, ${ }^{12 a}$ E. M. Gregores, ${ }^{12 b}$ C. Lagana, ${ }^{12 a}$ F. Marinho, ${ }^{12 a}$ P. G. Mercadante, ${ }^{12 b}$ S. F. Novaes, ${ }^{12 a}$ Sandra S. Padula, ${ }^{12 a}$ V. Genchev,${ }^{13, c}$ P. Iaydjiev, ${ }^{13, c}$ S. Piperov,${ }^{13}$ M. Rodozov,${ }^{13}$ S. Stoykova, ${ }^{13}$

G. Sultanov, ${ }^{13}$ V. Tcholakov, ${ }^{13}$ R. Trayanov, ${ }^{13}$ M. Vutova, ${ }^{13}$ A. Dimitrov, ${ }^{14}$ R. Hadjiiska, ${ }^{14}$ V. Kozhuharov, ${ }^{14}$ L. Litov, ${ }^{14}$ B. Pavlov, ${ }^{14}$ P. Petkov, ${ }^{14}$ J. G. Bian, ${ }^{15}$ G. M. Chen, ${ }^{15}$ H. S. Chen, ${ }^{15}$ C. H. Jiang, ${ }^{15}$ D. Liang, ${ }^{15}$ S. Liang, ${ }^{15}$

X. Meng, ${ }^{15}$ J. Tao,${ }^{15}$ J. Wang, ${ }^{15}$ X. Wang, ${ }^{15}$ Z. Wang, ${ }^{15}$ H. Xiao,${ }^{15}$ M. Xu, ${ }^{15}$ J. Zang,${ }^{15}$ Z. Zhang, ${ }^{15}$

C. Asawatangtrakuldee, ${ }^{16}$ Y. Ban,${ }^{16}$ Y. Guo, ${ }^{16}$ W. Li,${ }^{16}$ S. Liu,${ }^{16}$ Y. Mao, ${ }^{16}$ S. J. Qian, ${ }^{16}$ H. Teng, ${ }^{16}$ D. Wang, ${ }^{16}$

L. Zhang, ${ }^{16}$ W. Zou, ${ }^{16}$ C. Avila, ${ }^{17}$ C. A. Carrillo Montoya, ${ }^{17}$ J. P. Gomez,${ }^{17}$ B. Gomez Moreno, ${ }^{17}$

A. F. Osorio Oliveros,${ }^{17}$ J. C. Sanabria,${ }^{17}$ N. Godinovic, ${ }^{18}$ D. Lelas, ${ }^{18}$ R. Plestina, ${ }^{18, f}$ D. Polic,${ }^{18}$ I. Puljak, ${ }^{18, c}$ Z. Antunovic, ${ }^{19}$ M. Kovac, ${ }^{19}$ V. Brigljevic, ${ }^{20}$ S. Duric, ${ }^{20}$ K. Kadija, ${ }^{20}$ J. Luetic,${ }^{20}$ D. Mekterovic, ${ }^{20}$ S. Morovic, ${ }^{20}$ L. Tikvica, ${ }^{20}$ A. Attikis, ${ }^{21}$ M. Galanti, ${ }^{21}$ G. Mavromanolakis, ${ }^{21}$ J. Mousa, ${ }^{21}$ C. Nicolaou,${ }^{21}$ F. Ptochos,${ }^{21}$ P. A. Razis,${ }^{21}$

M. Finger, ${ }^{22}$ M. Finger, Jr., ${ }^{22}$ Y. Assran, ${ }^{23, g}$ S. Elgammal, ${ }^{23, h}$ A. Ellithi Kamel, ${ }^{23, i}$ A. M. Kuotb Awad, ${ }^{23, j}$

M. A. Mahmoud ${ }^{23, j}$ A. Radi,${ }^{23, k, 1}$ M. Kadastik,${ }^{24}$ M. Müntel,${ }^{24}$ M. Murumaa,${ }^{24}$ M. Raidal,${ }^{24}$ L. Rebane,${ }^{24}$ A. Tiko, ${ }^{24}$ P. Eerola,${ }^{25}$ G. Fedi, ${ }^{25}$ M. Voutilainen, ${ }^{25}$ J. Härkönen, ${ }^{26}$ A. Heikkinen, ${ }^{26}$ V. Karimäki, ${ }^{26}$ R. Kinnunen, ${ }^{26}$

M. J. Kortelainen, ${ }^{26}$ T. Lampén, ${ }^{26}$ K. Lassila-Perini, ${ }^{26}$ S. Lehti ${ }^{26}$ T. Lindén, ${ }^{26}$ P. Luukka, ${ }^{26}$ T. Mäenpääa,${ }^{26}$ T. Peltola ${ }^{26}$ E. Tuominen, ${ }^{26}$ J. Tuominiemi, ${ }^{26}$ E. Tuovinen,${ }^{26}$ D. Ungaro, ${ }^{26}$ L. Wendland ${ }^{26}$ A. Korpela, ${ }^{27}$ T. Tuuva, ${ }^{27}$ M. Besancon, ${ }^{28}$ S. Choudhury, ${ }^{28}$ F. Couderc,${ }^{28}$ M. Dejardin, ${ }^{28}$ D. Denegri, ${ }^{28}$ B. Fabbro, ${ }^{28}$ J. L. Faure, ${ }^{28}$ F. Ferri, ${ }^{28}$

S. Ganjour, ${ }^{28}$ A. Givernaud, ${ }^{28}$ P. Gras, ${ }^{28}$ G. Hamel de Monchenault, ${ }^{28}$ P. Jarry,${ }^{28}$ E. Locci, ${ }^{28}$ J. Malcles,${ }^{28}$ L. Millischer, ${ }^{28}$ A. Nayak, ${ }^{28}$ J. Rander, ${ }^{28}$ A. Rosowsky, ${ }^{28}$ M. Titov, ${ }^{28}$ S. Baffioni, ${ }^{29}$ F. Beaudette, ${ }^{29}$ L. Benhabib,${ }^{29}$ L. Bianchini ${ }^{29}$ M. Bluj, ${ }^{29, \mathrm{~m}}$ P. Busson, ${ }^{29}$ C. Charlot, ${ }^{29}$ N. Daci, ${ }^{29}$ T. Dahms,${ }^{29}$ M. Dalchenko, ${ }^{29}$ L. Dobrzynski, ${ }^{29}$ A. Florent, ${ }^{29}$ R. Granier de Cassagnac, ${ }^{29}$ M. Haguenauer, ${ }^{29}$ P. Miné, ${ }^{29}$ C. Mironov, ${ }^{29}$ I. N. Naranjo, ${ }^{29}$ M. Nguyen, ${ }^{29}$ C. Ochando, ${ }^{29}$ P. Paganini, ${ }^{29}$ D. Sabes,${ }^{29}$ R. Salerno, ${ }^{29}$ Y. Sirois,${ }^{29}$ C. Veelken,${ }^{29}$ A. Zabi, ${ }^{29}$ J.-L. Agram, ${ }^{30, n}$ J. Andrea, ${ }^{30}$ D. Bloch, ${ }^{30}$ D. Bodin, ${ }^{30}$ J.-M. Brom,${ }^{30}$ M. Cardaci, ${ }^{30}$ E. C. Chabert, ${ }^{30}$ C. Collard,${ }^{30}$ E. Conte, ${ }^{30, n}$ F. Drouhin, ${ }^{30, n}$ J.-C. Fontaine, ${ }^{30, n}$ D. Gelé, ${ }^{30}$ U. Goerlach, ${ }^{30}$ P. Juillot, ${ }^{30}$ A.-C. Le Bihan, ${ }^{30}$ P. Van Hove,${ }^{30}$ S. Beauceron, ${ }^{31}$ N. Beaupere, ${ }^{31}$ O. Bondu, ${ }^{31}$ G. Boudoul,,${ }^{31}$ S. Brochet,${ }^{31}$ J. Chasserat,${ }^{31}$ R. Chierici, ${ }^{31, c}$ D. Contardo, ${ }^{31}$ P. Depasse,${ }^{31}$ H. El Mamouni, ${ }^{31}$ J. Fay, ${ }^{31}$ S. Gascon, ${ }^{31}$ M. Gouzevitch,${ }^{31}$ B. Ille, ${ }^{31}$ T. Kurca, ${ }^{31}$ M. Lethuillier, ${ }^{31}$ L. Mirabito, ${ }^{31}$ S. Perries,${ }^{31}$ L. Sgandurra, ${ }^{31}$ V. Sordini, ${ }^{31}$ Y. Tschudi, ${ }^{31}$ P. Verdier, ${ }^{31}$ S. Viret, ${ }^{31}$ 
Z. Tsamalaidze, ${ }^{32, o}$ C. Autermann, ${ }^{33}$ S. Beranek, ${ }^{33}$ B. Calpas,${ }^{33}$ M. Edelhoff,,${ }^{33}$ L. Feld, ${ }^{33}$ N. Heracleous, ${ }^{33}$ O. Hindrichs, ${ }^{33}$ R. Jussen, ${ }^{33}$ K. Klein, ${ }^{33}$ J. Merz,${ }^{33}$ A. Ostapchuk,${ }^{33}$ A. Perieanu, ${ }^{33}$ F. Raupach, ${ }^{33}$ J. Sammet, ${ }^{33}$ S. Schael, ${ }^{33}$ D. Sprenger, ${ }^{33}$ H. Weber, ${ }^{33}$ B. Wittmer, ${ }^{33}$ V. Zhukov, ${ }^{33, p}$ M. Ata, ${ }^{34}$ J. Caudron, ${ }^{34}$ E. Dietz-Laursonn, ${ }^{34}$ D. Duchardt, ${ }^{34}$ M. Erdmann, ${ }^{34}$ R. Fischer, ${ }^{34}$ A. Güth, ${ }^{34}$ T. Hebbeker,${ }^{34}$ C. Heidemann, ${ }^{34}$ K. Hoepfner, ${ }^{34}$ D. Klingebiel, ${ }^{34}$ S. Knutzen, ${ }^{34}$ P. Kreuzer, ${ }^{34}$ M. Merschmeyer, ${ }^{34}$ A. Meyer, ${ }^{34}$ P. Millet, ${ }^{34}$ M. Olschewski, ${ }^{34}$ K. Padeken, ${ }^{34}$ P. Papacz,${ }^{34}$ H. Pieta, ${ }^{34}$ H. Reithler, ${ }^{34}$ S. A. Schmitz, ${ }^{34}$ F. Schneider, ${ }^{34}$ L. Sonnenschein,${ }^{34}$ J. Steggemann, ${ }^{34}$ D. Teyssier, ${ }^{34}$ S. Thüer, ${ }^{34}$ M. Weber, ${ }^{34}$ M. Bontenackels, ${ }^{35}$ V. Cherepanov, ${ }^{35}$ Y. Erdogan, ${ }^{35}$ G. Flügge, ${ }^{35}$ H. Geenen, ${ }^{35}$ M. Geisler,${ }^{35}$ W. Haj Ahmad, ${ }^{35}$ F. Hoehle, ${ }^{35}$ B. Kargoll, ${ }^{35}$ T. Kress,${ }^{35}$ Y. Kuessel, ${ }^{35}$ J. Lingemann, ${ }^{35, c}$ A. Nowack, ${ }^{35}$ I. M. Nugent,${ }^{35}$ L. Perchalla, ${ }^{35}$ O. Pooth,${ }^{35}$ P. Sauerland ${ }^{35}$ A. Stahl, ${ }^{35}$ M. Aldaya Martin, ${ }^{36}$ I. Asin, ${ }^{36}$ N. Bartosik, ${ }^{36}$ J. Behr, ${ }^{36}$ W. Behrenhoff, ${ }^{36}$ U. Behrens, ${ }^{36}$ M. Bergholz, ${ }^{36, q}$ A. Bethani ${ }^{36}$ K. Borras, ${ }^{36}$ A. Burgmeier,${ }^{36}$ A. Cakir,${ }^{36}$ L. Calligaris,${ }^{36}$ A. Campbell, ${ }^{36}$ E. Castro, ${ }^{36}$ F. Costanza, ${ }^{36}$ D. Dammann, ${ }^{36}$ C. Diez Pardos, ${ }^{36}$ T. Dorland, ${ }^{36}$ G. Eckerlin, ${ }^{36}$ D. Eckstein,,${ }^{36}$ G. Flucke, ${ }^{36}$ A. Geiser, ${ }^{36}$ I. Glushkov, ${ }^{36}$

P. Gunnellini, ${ }^{36}$ S. Habib, ${ }^{36}$ J. Hauk, ${ }^{36}$ G. Hellwig, ${ }^{36}$ H. Jung, ${ }^{36}$ M. Kasemann, ${ }^{36}$ P. Katsas, ${ }^{36}$ C. Kleinwort, ${ }^{36}$ H. Kluge, ${ }^{36}$ A. Knutsson, ${ }^{36}$ M. Krämer, ${ }^{36}$ D. Krücker, ${ }^{36}$ E. Kuznetsova, ${ }^{36}$ W. Lange, ${ }^{36}$ J. Leonard, ${ }^{36}$ W. Lohmann, ${ }^{36, q}$ B. Lutz,${ }^{36}$ R. Mankel, ${ }^{36}$ I. Marfin, ${ }^{36}$ M. Marienfeld ${ }^{36}$ I.-A. Melzer-Pellmann, ${ }^{36}$ A. B. Meyer, ${ }^{36}$ J. Mnich, ${ }^{36}$ A. Mussgiller, ${ }^{36}$ S. Naumann-Emme, ${ }^{36}$ O. Novgorodova, ${ }^{36}$ F. Nowak, ${ }^{36}$ J. Olzem, ${ }^{36}$ H. Perrey, ${ }^{36}$ A. Petrukhin, ${ }^{36}$ D. Pitzl, ${ }^{36}$ A. Raspereza,${ }^{36}$ P. M. Ribeiro Cipriano, ${ }^{36}$ C. Riedl, ${ }^{36}$ E. Ron, ${ }^{36}$ M. Rosin, ${ }^{36}$ J. Salfeld-Nebgen,${ }^{36}$ R. Schmidt, ${ }^{36, q}$ T. Schoerner-Sadenius, ${ }^{36}$ N. Sen, ${ }^{36}$ A. Spiridonov, ${ }^{36}$ M. Stein, ${ }^{36}$ R. Walsh, ${ }^{36}$ C. Wissing,,${ }^{36}$ V. Blobel,${ }^{37}$ H. Enderle, ${ }^{37}$ J. Erfle,${ }^{37}$ U. Gebbert, ${ }^{37}$ M. Görner, ${ }^{37}$ M. Gosselink, ${ }^{37}$ J. Haller, ${ }^{37}$ T. Hermanns, ${ }^{37}$ R. S. Höing, ${ }^{37}$ K. Kaschube, ${ }^{37}$ G. Kaussen, ${ }^{37}$ H. Kirschenmann, ${ }^{37}$ R. Klanner, ${ }^{37}$ J. Lange, ${ }^{37}$ T. Peiffer, ${ }^{37}$ N. Pietsch, ${ }^{37}$ D. Rathjens, ${ }^{37}$ C. Sander,${ }^{37}$ H. Schettler, ${ }^{37}$ P. Schleper, ${ }^{37}$ E. Schlieckau, ${ }^{37}$ A. Schmidt, ${ }^{37}$ M. Schröder, ${ }^{37}$ T. Schum, ${ }^{37}$ M. Seidel,${ }^{37}$ J. Sibille,${ }^{37, r}$ V. Sola,${ }^{37}$ H. Stadie, ${ }^{37}$ G. Steinbrück,${ }^{37}$ J. Thomsen,${ }^{37}$ L. Vanelderen,${ }^{37}$ C. Barth,${ }^{38}$ C. Baus, ${ }^{38}$ J. Berger, ${ }^{38}$ C. Böser, ${ }^{38}$ T. Chwalek, ${ }^{38}$ W. De Boer ${ }^{38}$ A. Descroix,${ }^{38}$ A. Dierlamm, ${ }^{38}$ M. Feindt, ${ }^{38}$ M. Guthoff, ${ }^{38, c}$ C. Hackstein, ${ }^{38}$ F. Hartmann, ${ }^{38, c}$ T. Hauth, ${ }^{38, c}$ M. Heinrich, ${ }^{38}$ H. Held, ${ }^{38}$ K. H. Hoffmann, ${ }^{38}$ U. Husemann, ${ }^{38}$ I. Katkov, ${ }^{38, p}$ J. R. Komaragiri, ${ }^{38}$ P. Lobelle Pardo ${ }^{38}$ D. Martschei, ${ }^{38}$ S. Mueller, ${ }^{38}$ Th. Müller, ${ }^{38}$ M. Niegel, ${ }^{38}$ A. Nürnberg, ${ }^{38}$ O. Oberst,${ }^{38}$ A. Oehler, ${ }^{38}$ J. Ott, ${ }^{38}$ G. Quast,${ }^{38}$ K. Rabbertz, ${ }^{38}$ F. Ratnikov, ${ }^{38}$ N. Ratnikova, ${ }^{38}$ S. Röcker, ${ }^{38}$ F.-P. Schilling, ${ }^{38}$ G. Schott, ${ }^{38}$ H. J. Simonis, ${ }^{38}$ F. M. Stober,${ }^{38}$ D. Troendle, ${ }^{38}$ R. Ulrich, ${ }^{38}$ J. Wagner-Kuhr, ${ }^{38}$ S. Wayand, ${ }^{38}$ T. Weiler, ${ }^{38}$ M. Zeise, ${ }^{38}$ G. Anagnostou, ${ }^{39}$ G. Daskalakis, ${ }^{39}$ T. Geralis, ${ }^{39}$

S. Kesisoglou, ${ }^{39}$ A. Kyriakis, ${ }^{39}$ D. Loukas, ${ }^{39}$ I. Manolakos, ${ }^{39}$ A. Markou, ${ }^{39}$ C. Markou, ${ }^{39}$ E. Ntomari, ${ }^{39}$

L. Gouskos, ${ }^{40}$ T. J. Mertzimekis,${ }^{40}$ A. Panagiotou, ${ }^{40}$ N. Saoulidou, ${ }^{40}$ I. Evangelou ${ }^{41}$ C. Foudas,${ }^{41}$ P. Kokkas, ${ }^{41}$ N. Manthos, ${ }^{41}$ I. Papadopoulos, ${ }^{41}$ G. Bencze,${ }^{42}$ C. Hajdu,${ }^{42}$ P. Hidas, ${ }^{42}$ D. Horvath, ${ }^{42, s}$ F. Sikler, ${ }^{42}$ V. Veszpremi ${ }^{42}$ G. Vesztergombi, ${ }^{42, t}$ A. J. Zsigmond, ${ }^{42}$ N. Beni, ${ }^{43}$ S. Czellar, ${ }^{43}$ J. Molnar, ${ }^{43}$ J. Palinkas, ${ }^{43}$ Z. Szillasi, ${ }^{43}$ J. Karancsi, ${ }^{44}$

P. Raics ${ }^{44}$ Z. L. Trocsanyi ${ }^{44}$ B. Ujvari, ${ }^{44}$ S. B. Beri, ${ }^{45}$ V. Bhatnagar, ${ }^{45}$ N. Dhingra, ${ }^{45}$ R. Gupta, ${ }^{45}$ M. Kaur, ${ }^{45}$ M. Z. Mehta, ${ }^{45}$ M. Mittal, ${ }^{45}$ N. Nishu,${ }^{45}$ L. K. Saini ${ }^{45}$ A. Sharma ${ }^{45}$ J. B. Singh,${ }^{45}$ Ashok Kumar, ${ }^{46}$ Arun Kumar ${ }^{46}$ S. Ahuja, ${ }^{46}$ A. Bhardwaj ${ }^{46}$ B. C. Choudhary, ${ }^{46}$ S. Malhotra, ${ }^{46}$ M. Naimuddin, ${ }^{46}$ K. Ranjan,${ }^{46}$ P. Saxena, ${ }^{46}$ V. Sharma, ${ }^{46}$ R. K. Shivpuri, ${ }^{46}$ S. Banerjee, ${ }^{47}$ S. Bhattacharya,${ }^{47}$ K. Chatterjee, ${ }^{47}$ S. Dutta, ${ }^{47}$ B. Gomber, ${ }^{47}$ Sa. Jain,${ }^{47}$ Sh. Jain, ${ }^{47}$ R. Khurana, ${ }^{47}$ A. Modak,${ }^{47}$ S. Mukherjee, ${ }^{47}$ D. Roy,${ }^{47}$ S. Sarkar, ${ }^{47}$ M. Sharan, ${ }^{47}$ A. Abdulsalam, ${ }^{48}$ D. Dutta, ${ }^{48}$ S. Kailas, ${ }^{48}$ V. Kumar, ${ }^{48}$ A. K. Mohanty, ${ }^{48, \mathrm{c}}$ L. M. Pant,${ }^{48}$ P. Shukla, ${ }^{48}$ T. Aziz, ${ }^{49}$ R. M. Chatterjee ${ }^{49}$ S. Ganguly, ${ }^{49}$ M. Guchait, ${ }^{49, \mathrm{u}}$ A. Gurtu, ${ }^{49, \mathrm{v}}$ M. Maity ${ }^{49, \mathrm{w}}$ G. Majumder, ${ }^{49}$ K. Mazumdar, ${ }^{49}$ G. B. Mohanty, ${ }^{49}$ B. Parida ${ }^{49}$ K. Sudhakar, ${ }^{49}$ N. Wickramage ${ }^{49}$ S. Banerjee, ${ }^{50}$ S. Dugad,${ }^{50}$ H. Arfaei,${ }^{51, \mathrm{x}}$ H. Bakhshiansohi, ${ }^{51}$ S. M. Etesami, ${ }^{51, y}$ A. Fahim, ${ }^{51, \mathrm{x}}$ M. Hashemi, ${ }^{51, \mathrm{z}}$ H. Hesari, ${ }^{51}$ A. Jafari, ${ }^{51}$ M. Khakzad, ${ }^{51}$

M. Mohammadi Najafabadi, ${ }^{51}$ S. Paktinat Mehdiabadi, ${ }^{51}$ B. Safarzadeh, ${ }^{51, a a}$ M. Zeinali, ${ }^{51}$ M. Abbrescia,,${ }^{52 a, 52 b}$ L. Barbone, ${ }^{52 a, 52 b}$ C. Calabria, ${ }^{52 a, 52 b, c}$ S. S. Chhibra, ${ }^{52 a, 52 b}$ A. Colaleo, ${ }^{52 a}$ D. Creanza, ${ }^{52 a, 52 c}$ N. De Filippis, ${ }^{52 a, 52 c, c}$ M. De Palma, ${ }^{52 \mathrm{a}, 52 \mathrm{~b}}$ L. Fiore, ${ }^{52 \mathrm{a}}$ G. Iaselli, ${ }^{52 \mathrm{a}, 52 \mathrm{c}}$ G. Maggi, ${ }^{52 \mathrm{a}, 52 \mathrm{c}}$ M. Maggi, ${ }^{52 \mathrm{a}}$ B. Marangelli, ${ }^{52 \mathrm{a}, 52 \mathrm{~b}}$ S. My, ${ }^{52 \mathrm{a}, 52 \mathrm{c}}$ S. Nuzzo, ${ }^{52 a, 52 b}$ N. Pacifico, ${ }^{52 a}$ A. Pompili, ${ }^{52 a, 52 b}$ G. Pugliese, ${ }^{52 a, 52 c}$ G. Selvaggi, ${ }^{52 a, 52 b}$ L. Silvestris, ${ }^{52 a}$ G. Singh, ${ }^{52 a, 52 b}$ R. Venditti, ${ }^{52 a, 52 b}$ P. Verwilligen, ${ }^{52 a}$ G. Zito, ${ }^{52 a}$ G. Abbiendi, ${ }^{53 a}$ A. C. Benvenuti, ${ }^{53 a}$ D. Bonacorsi, ${ }^{53 a, 53 b}$ S. Braibant-Giacomelli, ${ }^{53 a, 53 b}$ L. Brigliadori, ${ }^{53 a, 53 b}$ P. Capiluppi, ${ }^{53 a, 53 b}$ A. Castro, ${ }^{53 a, 53 b}$ F. R. Cavallo, ${ }^{53 a}$ M. Cuffiani, ${ }^{53 a, 53 b}$ G. M. Dallavalle, ${ }^{53 a}$ F. Fabbri, ${ }^{53 a}$ A. Fanfani, ${ }^{53 a, 53 b}$ D. Fasanella, ${ }^{53 a, 53 b}$ P. Giacomelli, ${ }^{53 a}$ C. Grandi, ${ }^{53 a}$ L. Guiducci, ${ }^{53 a, 53 b}$ S. Marcellini, ${ }^{53 a}$ G. Masetti, ${ }^{53 a}$ M. Meneghelli, ${ }^{53 a, 53 b, c}$ A. Montanari, ${ }^{53 a}$ F. L. Navarria, ${ }^{53 a, 53 b}$ F. Odorici, ${ }^{53 a}$ A. Perrotta ${ }^{53 a}$ F. Primavera, ${ }^{53 a, 53 b}$ A. M. Rossi, ${ }^{53 a, 53 b}$ T. Rovelli, ${ }^{53 a, 53 b}$ G. P. Siroli, ${ }^{53 a, 53 b}$ N. Tosi, ${ }^{53 a}$ R. Travaglini, ${ }^{53 a, 53 b}$ S. Albergo, ${ }^{54 a, 54 b}$ G. Cappello, ${ }^{54 a, 54 b}$ 
M. Chiorboli, ${ }^{54 a, 54 b}$ S. Costa, ${ }^{54 a, 54 b}$ R. Potenza ${ }^{54 a, 54 b}$ A. Tricomi, ${ }^{54 a, 54 b}$ C. Tuve,${ }^{54 a, 54 b}$ G. Barbagli, ${ }^{55 a}$ V. Ciulli, ${ }^{55 a, 55 b}$ C. Civinini, ${ }^{55 a}$ R. D’ Alessandro, ${ }^{55 a, 55 b}$ E. Focardi, ${ }^{55 a, 55 b}$ S. Frosali, ${ }^{55 a, 55 b}$ E. Gallo, ${ }^{55 a}$ S. Gonzi, ${ }^{55 a, 55 b}$ M. Meschini, ${ }^{55 a}$ S. Paoletti, ${ }^{55 a}$ G. Sguazzoni,${ }^{55 a}$ A. Tropiano, ${ }^{55 a, 55 b}$ L. Benussi, ${ }^{56}$ S. Bianco, ${ }^{56}$ S. Colafranceschi, ${ }^{56, b b}$ F. Fabbri, ${ }^{56}$ D. Piccolo, ${ }^{56}$ P. Fabbricatore, ${ }^{57 a}$ R. Musenich, ${ }^{57 a}$ S. Tosi, ${ }^{57 a, 57 b}$ A. Benaglia, ${ }^{58 a}$ F. De Guio, ${ }^{58 a, 58 b}$ L. Di Matteo, ${ }^{58 a, 58 b, c}$ S. Fiorendi, ${ }^{58 a, 58 b}$ S. Gennai, ${ }^{58 a, c}$ A. Ghezzi, ${ }^{58 a, 58 b}$ M. T. Lucchini, ${ }^{58 a, c}$ S. Malvezzi, ${ }^{58 a}$ R. A. Manzoni, ${ }^{58 a, 58 b}$ A. Martelli, ${ }^{58 a, 58 b}$ A. Massironi, ${ }^{58 a, 58 b}$ D. Menasce ${ }^{58 \mathrm{a}}$ L. Moroni, ${ }^{58 \mathrm{a}}$ M. Paganoni, ${ }^{58 \mathrm{a}, 58 \mathrm{~b}}$ D. Pedrini, ${ }^{58 a}$ S. Ragazzi, ${ }^{58 a, 58 b}$ N. Redaelli, ${ }^{58 a}$ T. Tabarelli de Fatis, ${ }^{58 a, 58 b}$ S. Buontempo ${ }^{59 a}$ N. Cavallo, ${ }^{59 a, 59 c}$ A. De Cosa ${ }^{59 a, 59 b, c}$ O. Dogangun,${ }^{59 a, 59 b}$ F. Fabozzi ${ }^{59 a, 59 c}$ A. O. M. Iorio, ${ }^{59 a, 59 b}$ L. Lista, ${ }^{59 a}$ S. Meola, ${ }^{59 a, 59 d, c c}$ M. Merola, ${ }^{59 a}$ P. Paolucci, ${ }^{59, c}$ P. Azzi,${ }^{60 a}$ N. Bacchetta, ${ }^{60 a, c}$ D. Bisello, ${ }^{60 a, 60 b}$ A. Branca, ${ }^{60 a, 60 b, c}$ R. Carlin, ${ }^{60 a, 60 b}$ P. Checchia ${ }^{60 a}$ T. Dorigo, ${ }^{60 a}$ F. Gasparini, ${ }^{60 a, 60 b}$ U. Gasparini, ${ }^{60 a, 60 b}$ A. Gozzelino, ${ }^{60 a}$ K. Kanishchev, ${ }^{60 a, 60 c}$ S. Lacaprara, ${ }^{60 \mathrm{a}}$ I. Lazzizzera, ${ }^{60 \mathrm{a}, 60 \mathrm{c}}$ M. Margoni ${ }^{60 \mathrm{a}, 60 \mathrm{~b}}$ A. T. Meneguzzo, ${ }^{60 \mathrm{a}, 60 \mathrm{~b}} \mathrm{~J}$. Pazzini, ${ }^{60 \mathrm{a}, 60 \mathrm{~b}}$ N. Pozzobon, ${ }^{60 \mathrm{a}, 60 \mathrm{~b}}$ P. Ronchese,${ }^{60 a, 60 b}$ F. Simonetto, ${ }^{60 a, 60 b}$ E. Torassa, ${ }^{60 \mathrm{a}} \mathrm{M}$. Tosi ${ }^{60 \mathrm{a}, 60 \mathrm{~b}}$ S. Vanini, ${ }^{60 \mathrm{a}, 60 \mathrm{~b}}$ P. Zotto, ${ }^{60 \mathrm{a}, 60 \mathrm{~b}}$
A. Zucchetta ${ }^{60 a, 60 b}$ G. Zumerle,${ }^{60 a, 60 b}$ M. Gabusi, ${ }^{61 a, 61 b}$ S. P. Ratti, ${ }^{61 \mathrm{a}, 61 \mathrm{~b}}$ C. Riccardi, ${ }^{61 \mathrm{a}, 61 \mathrm{~b}}$ P. Torre, ${ }^{61 \mathrm{a}, 61 \mathrm{~b}}$ P. Vitulo, ${ }^{61 a, 61 b}$ M. Biasini, ${ }^{62 a, 62 b}$ G. M. Bilei, ${ }^{62 a}$ L. Fanò, ${ }^{62 a, 62 b}$ P. Lariccia, ${ }^{62 a, 62 b}$ G. Mantovani, ${ }^{62 a, 62 b}$ M. Menichelli, ${ }^{62 a}$ A. Nappi ${ }^{62 a, 62 b, a}$ F. Romeo ${ }^{62 a, 62 b}$ A. Saha, ${ }^{62 a}$ A. Santocchia, ${ }^{62 a, 62 b}$ A. Spiezia, ${ }^{62 a, 62 b}$ S. Taroni, ${ }^{62 a, 62 b}$ P. Azzurri, ${ }^{63 a, 63 c}$ G. Bagliesi, ${ }^{63 a}$ J. Bernardini, ${ }^{63 a}$ T. Boccali, ${ }^{63 a}$ G. Broccolo, ${ }^{63 a, 63 c}$ R. Castaldi, ${ }^{63 a}$ R. T. D’Agnolo, ${ }^{63 a, 63 c, c}$ R. Dell'Orso, ${ }^{63 a}$ F. Fiori, ${ }^{63 a, 63 b, c}$ L. Foà,${ }^{63 a, 63 c}$ A. Giassi, ${ }^{63 a}$ A. Kraan, ${ }^{63 a}$ F. Ligabue,${ }^{63 a, 63 c}$ T. Lomtadze, ${ }^{63 a}$ L. Martini, ${ }^{63 a, d d}$ A. Messineo ${ }^{63 a, 63 b}$ F. Palla, ${ }^{63 a}$ A. Rizzi, ${ }^{63 a, 63 b}$ A. T. Serban, ${ }^{63 a, e e}$ P. Spagnolo, ${ }^{63 a}$ P. Squillacioti, ${ }^{63 a, c}$ R. Tenchini, ${ }^{63 a}$ G. Tonelli, ${ }^{63 a, 63 b}$ A. Venturi, ${ }^{63 a}$ P. G. Verdini, ${ }^{63 a}$ L. Barone,${ }^{64 a, 64 b}$ F. Cavallari, ${ }^{64 a}$

D. Del Re,${ }^{64 a, 64 b}$ M. Diemoz, ${ }^{64 a}$ C. Fanelli, ${ }^{64 a, 64 b}$ M. Grassi, ${ }^{64 a, 64 b, c}$ E. Longo, ${ }^{64 a, 64 b}$ P. Meridiani, ${ }^{64 a, c}$

F. Micheli, ${ }^{64 a, 64 b}$ S. Nourbakhsh, ${ }^{64 a, 64 b}$ G. Organtini, ${ }^{64 a, 64 b}$ R. Paramatti ${ }^{64 a}$ S. Rahatlou, ${ }^{64 a, 64 b}$ L. Soffi, ${ }^{64 a, 64 b}$ N. Amapane, ${ }^{65 a, 65 b}$ R. Arcidiacono, ${ }^{65 a, 65 c}$ S. Argiro, ${ }^{65 a, 65 b}$ M. Arneodo,${ }^{65 a, 65 c}$ C. Biino, ${ }^{65 a}$ N. Cartiglia, ${ }^{65 a}$ S. Casasso,${ }^{65 a, 65 b}$ M. Costa, ${ }^{65 a, 65 b}$ N. Demaria, ${ }^{65 a}$ C. Mariotti, ${ }^{65 a, c}$ S. Maselli, ${ }^{65 a}$ E. Migliore, ${ }^{65 a, 65 b}$ V. Monaco,${ }^{65 a, 65 b}$ M. Musich, ${ }^{65 a, c}$ M. M. Obertino, ${ }^{65 a, 65 c}$ N. Pastrone, ${ }^{65 a}$ M. Pelliccioni, ${ }^{65 a}$ A. Potenza, ${ }^{65 a, 65 b}$ A. Romero, ${ }^{65 a, 65 b}$ M. Ruspa ${ }^{65 a, 65 c}$ R. Sacchi, ${ }^{65 a, 65 b}$ A. Solano, ${ }^{65 a, 65 b}$ A. Staiano, ${ }^{65 a}$ S. Belforte, ${ }^{66 a}$ V. Candelise, ${ }^{66 a, 66 b}$ M. Casarsa ${ }^{66 a}$ F. Cossutti, ${ }^{66 a, c}$ G. Della Ricca, ${ }^{66 a, 66 b}$ B. Gobbo, ${ }^{66 a}$ M. Marone, ${ }^{66 a, 66 b, c}$ D. Montanino, ${ }^{66 a, 66 b}$ A. Penzo, ${ }^{66 a}$

A. Schizzi, ${ }^{66 a, 66 b}$ T. Y. Kim, ${ }^{67}$ S. K. Nam, ${ }^{67}$ S. Chang, ${ }^{68}$ D. H. Kim, ${ }^{68}$ G. N. Kim, ${ }^{68}$ D. J. Kong, ${ }^{68}$ Y. D. Oh, ${ }^{68}$ H. Park, ${ }^{68}$ D. C. Son, ${ }^{68}$ J. Y. Kim, ${ }^{69}$ Zero J. Kim, ${ }^{69}$ S. Song, ${ }^{69}$ S. Choi, ${ }^{70}$ D. Gyun, ${ }^{70}$ B. Hong, ${ }^{70}$ M. Jo, ${ }^{70}$ H. Kim, ${ }^{70}$ T. J. Kim, ${ }^{70}$ K. S. Lee, ${ }^{70}$ D. H. Moon, ${ }^{70}$ S. K. Park, ${ }^{70}$ Y. Roh,${ }^{70}$ M. Choi, ${ }^{71}$ J. H. Kim,${ }^{71}$ C. Park, ${ }^{71}$ I. C. Park, ${ }^{71}$ S. Park,${ }^{71}$ G. Ryuu ${ }^{71}$ Y. Choi, ${ }^{72}$ Y. K. Choi,${ }^{72}$ J. Goh, ${ }^{72}$ M. S. Kim,${ }^{72}$ E. Kwon, ${ }^{72}$ B. Lee, ${ }^{72}$ J. Lee,${ }^{72}$ S. Lee,${ }^{72}$ H. Seo, ${ }^{72}$ I. Yu ${ }^{72}$ M. J. Bilinskas, ${ }^{73}$ I. Grigelionis, ${ }^{73}$ M. Janulis, ${ }^{73}$ A. Juodagalvis,${ }^{73}$ H. Castilla-Valdez,${ }^{74}$ E. De La Cruz-Burelo,${ }^{74}$ I. Heredia-de La Cruz ${ }^{74}$ R. Lopez-Fernandez, ${ }^{74}$ J. Martínez-Ortega ${ }^{74}$

A. Sanchez-Hernandez, ${ }^{74}$ L. M. Villasenor-Cendejas, ${ }^{74}$ S. Carrillo Moreno, ${ }^{75}$ F. Vazquez Valencia, ${ }^{75}$ H. A. Salazar Ibarguen, ${ }^{76}$ E. Casimiro Linares, ${ }^{77}$ A. Morelos Pineda,${ }^{77}$ M. A. Reyes-Santos,${ }^{77}$ D. Krofcheck,${ }^{78}$ A. J. Bell, ${ }^{79}$ P. H. Butler, ${ }^{79}$ R. Doesburg, ${ }^{79}$ S. Reucroft, ${ }^{79}$ H. Silverwood, ${ }^{79}$ M. Ahmad, ${ }^{80}$ M. I. Asghar, ${ }^{80}$ J. Butt, ${ }^{80}$ H. R. Hoorani ${ }^{80}$ S. Khalid, ${ }^{80}$ W. A. Khan ${ }^{80}$ T. Khurshid, ${ }^{80}$ S. Qazi, ${ }^{80}$ M. A. Shah,${ }^{80}$ M. Shoaib,${ }^{80}$ H. Bialkowska, ${ }^{81}$ B. Boimska, ${ }^{81}$ T. Frueboes,${ }^{81}$ M. Górski, ${ }^{81}$ M. Kazana,${ }^{81}$ K. Nawrocki, ${ }^{81}$ K. Romanowska-Rybinska, ${ }^{81}$ M. Szleper, ${ }^{81}$ G. Wrochna ${ }^{81}$ P. Zalewski, ${ }^{81}$ G. Brona,${ }^{82}$ K. Bunkowski, ${ }^{82}$ M. Cwiok, ${ }^{82}$ W. Dominik, ${ }^{82}$ K. Doroba, ${ }^{82}$

A. Kalinowski, ${ }^{82}$ M. Konecki, ${ }^{82}$ J. Krolikowski, ${ }^{82}$ M. Misiura ${ }^{82}$ W. Wolszczak, ${ }^{82}$ N. Almeida ${ }^{83}$ P. Bargassa ${ }^{83}$

A. David ${ }^{83}$ P. Faccioli, ${ }^{83}$ P. G. Ferreira Parracho,${ }^{83}$ M. Gallinaro, ${ }^{83}$ J. Seixas, ${ }^{83}$ J. Varela, ${ }^{83}$ P. Vischia, ${ }^{83}$

I. Belotelov, ${ }^{84}$ P. Bunin, ${ }^{84}$ I. Golutvin, ${ }^{84}$ I. Gorbunov, ${ }^{84}$ A. Kamenev, ${ }^{84}$ V. Karjavin, ${ }^{84}$ G. Kozlov,${ }^{84}$ A. Lanev, ${ }^{84}$ A. Malakhov ${ }^{84}$ P. Moisenz, ${ }^{84}$ V. Palichik, ${ }^{84}$ V. Perelygin,${ }^{84}$ M. Savina,${ }^{84}$ S. Shmatov, ${ }^{84}$ V. Smirnov, ${ }^{84}$ A. Volodko, ${ }^{84}$ A. Zarubin, ${ }^{84}$ S. Evstyukhin, ${ }^{85}$ V. Golovtsov, ${ }^{85}$ Y. Ivanov, ${ }^{85}$ V. Kim,,${ }^{85}$ P. Levchenko, ${ }^{85}$ V. Murzin, ${ }^{85}$ V. Oreshkin, ${ }^{85}$ I. Smirnov, ${ }^{85}$ V. Sulimov, ${ }^{85}$ L. Uvarov, ${ }^{85}$ S. Vavilov, ${ }^{85}$ A. Vorobyev,${ }^{85}$ An. Vorobyev, ${ }^{85}$ Yu. Andreev, ${ }^{86}$ A. Dermenev, ${ }^{86}$ S. Gninenko, ${ }^{86}$ N. Golubev, ${ }^{86}$ M. Kirsanov, ${ }^{86}$ N. Krasnikov, ${ }^{86}$ V. Matveev, ${ }^{86}$ A. Pashenkov, ${ }^{86}$ D. Tlisov, ${ }^{86}$ A. Toropin, ${ }^{86}$ V. Epshteyn, ${ }^{87}$ M. Erofeeva, ${ }^{87}$ V. Gavrilov, ${ }^{87}$ M. Kossov, ${ }^{87}$ N. Lychkovskaya, ${ }^{87}$ V. Popov ${ }^{87}$ G. Safronov, ${ }^{87}$ S. Semenov, ${ }^{87}$ I. Shreyber, ${ }^{87}$ V. Stolin, ${ }^{87}$ E. Vlasov, ${ }^{87}$ A. Zhokin,${ }^{87}$ V. Andreev, ${ }^{88}$ M. Azarkin, ${ }^{88}$ I. Dremin, ${ }^{88}$ M. Kirakosyan, ${ }^{88}$ A. Leonidov, ${ }^{88}$ G. Mesyats, ${ }^{88}$ S. V. Rusakov,${ }^{88}$ A. Vinogradov, ${ }^{88}$ A. Belyaev, ${ }^{89}$ E. Boos ${ }^{89}$ M. Dubinin, ${ }^{89, e}$ L. Dudko, ${ }^{89}$ A. Ershov, ${ }^{89}$ A. Gribushin, ${ }^{89}$ V. Klyukhin, ${ }^{89}$ O. Kodolova, ${ }^{89}$ I. Lokhtin,${ }^{89}$

A. Markina ${ }^{89}$ S. Obraztsov, ${ }^{89}$ M. Perfilov, ${ }^{89}$ S. Petrushanko, ${ }^{89}$ A. Popov, ${ }^{89}$ L. Sarycheva, ${ }^{89, a}$ V. Savrin, ${ }^{89}$ A. Snigirev, ${ }^{89}$ I. Azhgirey, ${ }^{90}$ I. Bayshev,${ }^{90}$ S. Bitioukov, ${ }^{90}$ V. Grishin, ${ }^{90, c}$ V. Kachanov, ${ }^{90}$ D. Konstantinov,${ }^{90}$ 
V. Krychkine,${ }^{90}$ V. Petrov, ${ }^{90}$ R. Ryutin,,${ }^{90}$ A. Sobol, ${ }^{90}$ L. Tourtchanovitch,,${ }^{90}$ S. Troshin,${ }^{90}$ N. Tyurin, ${ }^{90}$ A. Uzunian,,${ }^{90}$ A. Volkov, ${ }^{90}$ P. Adzic,${ }^{91, f f}$ M. Djordjevic, ${ }^{91}$ M. Ekmedzic, ${ }^{91}$ D. Krpic, ${ }^{91, f f}$ J. Milosevic, ${ }^{91}$ M. Aguilar-Benitez, ${ }^{92}$ J. Alcaraz Maestre, ${ }^{92}$ P. Arce, ${ }^{92}$ C. Battilana, ${ }^{92}$ E. Calvo, ${ }^{92}$ M. Cerrada, ${ }^{92}$ M. Chamizo Llatas, ${ }^{92}$ N. Colino, ${ }^{92}$ B. De La Cruz, ${ }^{92}$ A. Delgado Peris, ${ }^{92}$ D. Domínguez Vázquez, ${ }^{92}$ C. Fernandez Bedoya,${ }^{92}$ J. P. Fernández Ramos, ${ }^{92}$ A. Ferrando ${ }^{92}$ J. Flix,${ }^{92}$ M. C. Fouz, ${ }^{92}$ P. Garcia-Abia, ${ }^{92}$ O. Gonzalez Lopez, ${ }^{92}$ S. Goy Lopez, ${ }^{92}$ J. M. Hernandez, ${ }^{92}$ M. I. Josa, ${ }^{92}$ G. Merino, ${ }^{92}$ J. Puerta Pelayo, ${ }^{92}$ A. Quintario Olmeda, ${ }^{92}$ I. Redondo, ${ }^{92}$ L. Romero, ${ }^{92}$ J. Santaolalla, ${ }^{92}$ M. S. Soares, ${ }^{92}$ C. Willmott, ${ }^{92}$ C. Albajar, ${ }^{93}$ G. Codispoti, ${ }^{93}$ J. F. de Trocóniz, ${ }^{93}$ H. Brun, ${ }^{94}$ J. Cuevas, ${ }^{94}$

J. Fernandez Menendez, ${ }^{94}$ S. Folgueras,${ }^{94}$ I. Gonzalez Caballero, ${ }^{94}$ L. Lloret Iglesias, ${ }^{94}$ J. Piedra Gomez,,${ }^{94}$

J. A. Brochero Cifuentes, ${ }^{95}$ I. J. Cabrillo, ${ }^{95}$ A. Calderon, ${ }^{95}$ S. H. Chuang,,${ }^{95}$ J. Duarte Campderros, ${ }^{95}$ M. Felcini, ${ }^{95, g g}$ M. Fernandez, ${ }^{95}$ G. Gomez, ${ }^{95}$ J. Gonzalez Sanchez, ${ }^{95}$ A. Graziano, ${ }^{95}$ C. Jorda ${ }^{95}$ A. Lopez Virto, ${ }^{95}$ J. Marco, ${ }^{95}$ R. Marco, ${ }^{95}$ C. Martinez Rivero, ${ }^{95}$ F. Matorras, ${ }^{95}$ F. J. Munoz Sanchez, ${ }^{95}$ T. Rodrigo, ${ }^{95}$ A. Y. Rodríguez-Marrero, ${ }^{95}$ A. Ruiz-Jimeno, ${ }^{95}$ L. Scodellaro, ${ }^{95}$ I. Vila ${ }^{95}$ R. Vilar Cortabitarte, ${ }^{95}$ D. Abbaneo,${ }^{96}$ E. Auffray, ${ }^{96}$ G. Auzinger,${ }^{96}$ M. Bachtis, ${ }^{96}$ P. Baillon, ${ }^{96}$ A. H. Ball, ${ }^{96}$ D. Barney, ${ }^{96}$ J. F. Benitez,${ }^{96}$ C. Bernet,${ }^{96, f}$ G. Bianchi, ${ }^{96}$ P. Bloch,${ }^{96}$ A. Bocci, ${ }^{96}$ A. Bonato, ${ }^{96}$ C. Botta, ${ }^{96}$ H. Breuker, ${ }^{96}$ T. Camporesi, ${ }^{96}$ G. Cerminara,${ }^{96}$ T. Christiansen, ${ }^{96}$ J. A. Coarasa Perez, ${ }^{96}$ D. D'Enterria, ${ }^{96}$ A. Dabrowski, ${ }^{96}$ A. De Roeck, ${ }^{96}$ S. Di Guida,${ }^{96}$ M. Dobson, ${ }^{96}$ N. Dupont-Sagorin, ${ }^{96}$ A. Elliott-Peisert, ${ }^{96}$ B. Frisch, ${ }^{96}$ W. Funk, ${ }^{96}$ G. Georgiou, ${ }^{96}$ M. Giffels,,${ }^{96}$ D. Gigi, ${ }^{96}$ K. Gill, ${ }^{96}$ D. Giordano,${ }^{96}$ M. Girone,${ }^{96}$ M. Giunta, ${ }^{96}$ F. Glege,${ }^{96}$ R. Gomez-Reino Garrido,${ }^{96}$ P. Govoni, ${ }^{96}$ S. Gowdy, ${ }^{96}$ R. Guida ${ }^{96}$ J. Hammer, ${ }^{96}$ M. Hansen, ${ }^{96}$ P. Harris,${ }^{96}$ C. Hartl, ${ }^{96}$ J. Harvey,${ }^{96}$ B. Hegner, ${ }^{96}$ A. Hinzmann, ${ }^{96}$ V. Innocente, ${ }^{96}$ P. Janot, ${ }^{96}$ K. Kaadze,${ }^{96}$ E. Karavakis, ${ }^{96}$ K. Kousouris, ${ }^{96}$ P. Lecoq, ${ }^{96}$ Y.-J. Lee, ${ }^{96}$ P. Lenzi,,${ }^{96}$ C. Lourenço, ${ }^{96}$ N. Magini, ${ }^{96}$ T. Mäki,${ }^{96}$ M. Malberti, ${ }^{96}$ L. Malgeri, ${ }^{96}$ M. Mannelli, ${ }^{96}$ L. Masetti, ${ }^{96}$ F. Meijers, ${ }^{96}$ S. Mersi, ${ }^{96}$ E. Meschi, ${ }^{96}$ R. Moser, ${ }^{96}$ M. Mulders, ${ }^{96}$ P. Musella, ${ }^{96}$ E. Nesvold, ${ }^{96}$ L. Orsini, ${ }^{96}$ E. Palencia Cortezon, ${ }^{96}$ E. Perez,${ }^{96}$ L. Perrozzi,${ }^{96}$ A. Petrilli, ${ }^{96}$ A. Pfeiffer, ${ }^{96}$ M. Pierini,${ }^{96}$ M. Pimiä, ${ }^{96}$ D. Piparo, ${ }^{96}$ G. Polese,${ }^{96}$ L. Quertenmont, ${ }^{96}$ A. Racz, ${ }^{96}$ W. Reece, ${ }^{96}$ J. Rodrigues Antunes, ${ }^{96}$ G. Rolandi, ${ }^{96, \text { hh }}$ C. Rovelli, ${ }^{96, i i}$ M. Rovere, ${ }^{96}$ H. Sakulin, ${ }^{96}$ F. Santanastasio, ${ }^{96}$ C. Schäfer, ${ }^{96}$ C. Schwick,${ }^{96}$ I. Segoni, ${ }^{96}$ S. Sekmen, ${ }^{96}$ A. Sharma, ${ }^{96}$ P. Siegrist, ${ }^{96}$ P. Silva, ${ }^{96}$ M. Simon, ${ }^{96}$ P. Sphicas, ${ }^{96, j j}$ D. Spiga, ${ }^{96}$ A. Tsirou, ${ }^{96}$ G. I. Veres, ${ }^{96, t}$ J. R. Vlimant,${ }^{96}$ H. K. Wöhri, ${ }^{96}$ S. D. Worm, ${ }^{96, \mathrm{kk}}$ W. D. Zeuner, ${ }^{96}$ W. Bertl, ${ }^{97}$ K. Deiters,${ }^{97}$ W. Erdmann, ${ }^{97}$ K. Gabathuler, ${ }^{97}$ R. Horisberger, ${ }^{97}$ Q. Ingram, ${ }^{97}$ H. C. Kaestli, ${ }^{97}$ S. König, ${ }^{97}$ D. Kotlinski, ${ }^{97}$ U. Langenegger, ${ }^{97}$ F. Meier, ${ }^{97}$ D. Renker, ${ }^{97}$ T. Rohe, ${ }^{97}$ F. Bachmair, ${ }^{98}$ L. Bäni, ${ }^{98}$ P. Bortignon, ${ }^{98}$ M. A. Buchmann, ${ }^{98}$ B. Casal,${ }^{98}$ N. Chanon, ${ }^{98}$ A. Deisher, ${ }^{98}$ G. Dissertori, ${ }^{98}$ M. Dittmar, ${ }^{98}$ M. Donegà, ${ }^{98}$ M. Dünser, ${ }^{98}$ P. Eller, ${ }^{98}$ J. Eugster, ${ }^{98}$ K. Freudenreich, ${ }^{98}$ C. Grab,${ }^{98}$ D. Hits,${ }^{98}$ P. Lecomte, ${ }^{98}$ W. Lustermann, ${ }^{98}$ A. C. Marini, ${ }^{98}$ P. Martinez Ruiz del Arbol,${ }^{98}$ N. Mohr,${ }^{98}$ F. Moortgat,${ }^{98}$ C. Nägeli, ${ }^{98,11}$ P. Nef, ${ }^{98}$ F. Nessi-Tedaldi, ${ }^{98}$ F. Pandolfi, ${ }^{98}$ L. Pape, ${ }^{98}$ F. Pauss, ${ }^{98}$ M. Peruzzi, ${ }^{98}$ F. J. Ronga,${ }^{98}$ M. Rossini, ${ }^{98}$ L. Sala, ${ }^{98}$ A. K. Sanchez, ${ }^{98}$ A. Starodumov, ${ }^{98, m m}$ B. Stieger, ${ }^{98}$ M. Takahashi, ${ }^{98}$ L. Tauscher, ${ }^{98, a}$ A. Thea,${ }^{98}$ K. Theofilatos, ${ }^{98}$ D. Treille, ${ }^{98}$ C. Urscheler, ${ }^{98}$ R. Wallny,${ }^{98}$ H. A. Weber, ${ }^{98}$ L. Wehrli, ${ }^{98}$ C. Amsler, ${ }^{99, n n}$ V. Chiochia, ${ }^{99}$ S. De Visscher, ${ }^{99}$ C. Favaro, ${ }^{99}$ M. Ivova Rikova, ${ }^{99}$ B. Kilminster, ${ }^{99}$ B. Millan Mejias, ${ }^{99}$ P. Otiougova, ${ }^{99}$ P. Robmann, ${ }^{99}$ H. Snoek, ${ }^{99}$ S. Tupputi, ${ }^{99}$ M. Verzetti, ${ }^{99}$ Y. H. Chang, ${ }^{100}$ K. H. Chen, ${ }^{100}$ C. Ferro, ${ }^{100}$ C. M. Kuo, ${ }^{100}$ S. W. Li, ${ }^{100}$ W. Lin, ${ }^{100}$ Y. J. Lu, ${ }^{100}$ A. P. Singh, ${ }^{100}$ R. Volpe,${ }^{100}$ S. S. Yu, ${ }^{100}$ P. Bartalini, ${ }^{101}$ P. Chang, ${ }^{101}$ Y. H. Chang, ${ }^{101}$ Y. W. Chang, ${ }^{101}$ Y. Chao, ${ }^{101}$ K. F. Chen, ${ }^{101}$ C. Dietz, ${ }^{101}$ U. Grundler,${ }^{101}$ W.-S. Hou, ${ }^{101}$ Y. Hsiung,,${ }^{101}$ K. Y. Kao ${ }^{101}$ Y. J. Lei, ${ }^{101}$ R.-S. Lu ${ }^{101}$ D. Majumder, ${ }^{101}$ E. Petrakou, ${ }^{101}$ X. Shi, ${ }^{101}$ J. G. Shiu, ${ }^{101}$ Y. M. Tzeng, ${ }^{101}$ X. Wan, ${ }^{101}$ M. Wang,${ }^{101}$ B. Asavapibhop, ${ }^{102}$ E. Simili, ${ }^{102}$ N. Srimanobhas, ${ }^{102}$ N. Suwonjandee, ${ }^{102}$ A. Adiguzel,${ }^{103}$ M. N. Bakirci, ${ }^{103,{ }^{\circ o}}$ S. Cerci, ${ }^{103, p p}$ C. Dozen, ${ }^{103}$ I. Dumanoglu, ${ }^{103}$ E. Eskut, ${ }^{103}$ S. Girgis, ${ }^{103}$ G. Gokbulut, ${ }^{103}$ E. Gurpinar, ${ }^{103}$ I. Hos, ${ }^{103}$ E. E. Kangal,${ }^{103}$ T. Karaman, ${ }^{103}$ G. Karapinar, ${ }^{103, q 9}$ A. Kayis Topaksu, ${ }^{103}$ G. Onengut,${ }^{103}$ K. Ozdemir, ${ }^{103}$ S. Ozturk, ${ }^{103, \text { rr }}$ A. Polatoz,${ }^{103}$ K. Sogut, ${ }^{103, \text { ss }}$ D. Sunar Cerci, ${ }^{103, p p}$ B. Tali, ${ }^{103, p p}$ H. Topakli, ${ }^{103, o o}$ L. N. Vergili, ${ }^{103}$ M. Vergili, ${ }^{103}$ I. V. Akin,,${ }^{104}$ T. Aliev, ${ }^{104}$ B. Bilin, ${ }^{104}$ S. Bilmis, ${ }^{104}$ M. Deniz, ${ }^{104}$ H. Gamsizkan, ${ }^{104}$ A. M. Guler, ${ }^{104}$ K. Ocalan, ${ }^{104}$ A. Ozpineci, ${ }^{104}$ M. Serin, ${ }^{104}$ R. Sever, ${ }^{104}$ U. E. Surat,${ }^{104}$ M. Yalvac, ${ }^{104}$ E. Yildirim, ${ }^{104}$ M. Zeyrek, ${ }^{104}$ E. Gülmez, ${ }^{105}$ B. Isildak, ${ }^{105, t t}$ M. Kaya, ${ }^{105, \text { uu }}$ O. Kaya, ${ }^{105, \text { uu }}$ S. Ozkorucuklu, ${ }^{105, v v}$ N. Sonmez, ${ }^{105, w w}$ H. Bahtiyar, ${ }^{106, x x}$ E. Barlas, ${ }^{106}$ K. Cankocak, ${ }^{106}$ Y. O. Günaydin, ${ }^{106, y y}$ F. I. Vardarl,${ }^{106}$ M. Yücel, ${ }^{106}$ L. Levchuk, ${ }^{107}$ J. J. Brooke, ${ }^{108}$ E. Clement, ${ }^{108}$ D. Cussans, ${ }^{108}$ H. Flacher, ${ }^{108}$ R. Frazier, ${ }^{108}$ J. Goldstein, ${ }^{108}$ M. Grimes,${ }^{108}$ G. P. Heath, ${ }^{108}$ H. F. Heath, ${ }^{108}$ L. Kreczko, ${ }^{108}$ S. Metson, ${ }^{108}$ D. M. Newbold ${ }^{108, k k}$ K. Nirunpong, ${ }^{108}$ A. Poll, ${ }^{108}$ S. Senkin, ${ }^{108}$ V. J. Smith, ${ }^{108}$ T. Williams, ${ }^{108}$ L. Basso, ${ }^{109, z z}$ K. W. Bell,${ }^{109}$ A. Belyaev, ${ }^{109, z z}$ C. Brew, ${ }^{109}$ R. M. Brown, ${ }^{109}$ D. J. A. Cockerill, ${ }^{109}$ J. A. Coughlan, ${ }^{109}$ K. Harder, ${ }^{109}$ S. Harper, ${ }^{109}$ J. Jackson, ${ }^{109}$ B. W. Kennedy, ${ }^{109}$ E. Olaiya, ${ }^{109}$ D. Petyt, ${ }^{109}$ B. C. Radburn-Smith, ${ }^{109}$ C. H. Shepherd-Themistocleous, ${ }^{109}$ I. R. Tomalin, ${ }^{109}$ 
W. J. Womersley, ${ }^{109}$ R. Bainbridge, ${ }^{110}$ G. Ball, ${ }^{110}$ R. Beuselinck, ${ }^{110}$ O. Buchmuller, ${ }^{110}$ D. Colling,,${ }^{110}$ N. Cripps, ${ }^{110}$ M. Cutajar, ${ }^{110}$ P. Dauncey, ${ }^{110}$ G. Davies,${ }^{110}$ M. Della Negra, ${ }^{110}$ W. Ferguson, ${ }^{110}$ J. Fulcher, ${ }^{110}$ D. Futyan,${ }^{110}$

A. Gilbert, ${ }^{110}$ A. Guneratne Bryer, ${ }^{110}$ G. Hall, ${ }^{110}$ Z. Hatherell, ${ }^{110}$ J. Hays,,${ }^{110}$ G. Iles, ${ }^{110}$ M. Jarvis, ${ }^{110}$ G. Karapostoli, ${ }^{110}$ M. Kenzie, ${ }^{110}$ L. Lyons, ${ }^{110}$ A.-M. Magnan, ${ }^{110}$ J. Marrouche, ${ }^{110}$ B. Mathias, ${ }^{110}$ R. Nandi, ${ }^{110}$ J. Nash, ${ }^{110}$ A. Nikitenko, ${ }^{110, \mathrm{~mm}}$ J. Pela, ${ }^{110}$ M. Pesaresi, ${ }^{110}$ K. Petridis, ${ }^{110}$ M. Pioppi, ${ }^{110, \text { aaa }}$ D. M. Raymond,${ }^{110}$

S. Rogerson, ${ }^{110}$ A. Rose,${ }^{110}$ C. Seez, ${ }^{110}$ P. Sharp, ${ }^{110, a}$ A. Sparrow, ${ }^{110}$ M. Stoye, ${ }^{110}$ A. Tapper, ${ }^{110}$

M. Vazquez Acosta, ${ }^{110}$ T. Virdee, ${ }^{110} \mathrm{~S}$. Wakefield, ${ }^{110}$ N. Wardle, ${ }^{110}$ T. Whyntie,${ }^{110}$ M. Chadwick,,${ }^{11}$ J.E. Cole, ${ }^{111}$ P. R. Hobson, ${ }^{111}$ A. Khan, ${ }^{111}$ P. Kyberd, ${ }^{111}$ D. Leggat, ${ }^{111}$ D. Leslie,,${ }^{111}$ W. Martin, ${ }^{111}$ I. D. Reid, ${ }^{111}$ P. Symonds, ${ }^{111}$ L. Teodorescu ${ }^{111}$ M. Turner,${ }^{111}$ K. Hatakeyama, ${ }^{112}$ H. Liu, ${ }^{112}$ T. Scarborough, ${ }^{112}$ O. Charaf, ${ }^{113}$ S. I. Cooper, ${ }^{113}$ C. Henderson, ${ }^{113}$ P. Rumerio, ${ }^{113}$ A. Avetisyan, ${ }^{114}$ T. Bose,${ }^{114}$ C. Fantasia, ${ }^{114}$ A. Heister, ${ }^{114}$ P. Lawson, ${ }^{114}$ D. Lazic, ${ }^{114}$

J. Rohlf, ${ }^{114}$ D. Sperka, ${ }^{114}$ J. St. John, ${ }^{114}$ L. Sulak, ${ }^{114}$ J. Alimena, ${ }^{115}$ S. Bhattacharya, ${ }^{115}$ G. Christopher, ${ }^{115}$

D. Cutts ${ }^{115}$ Z. Demiragli, ${ }^{115}$ A. Ferapontov, ${ }^{115}$ A. Garabedian, ${ }^{115}$ U. Heintz, ${ }^{115}$ S. Jabeen, ${ }^{115}$ G. Kukartsev, ${ }^{115}$ E. Laird, ${ }^{115}$ G. Landsberg, ${ }^{115}$ M. Luk, ${ }^{115}$ M. Narain, ${ }^{115}$ M. Segala, ${ }^{115}$ T. Sinthuprasith, ${ }^{115}$ T. Speer, ${ }^{115}$ R. Breedon, ${ }^{116}$ G. Breto, ${ }^{116}$ M. Calderon De La Barca Sanchez, ${ }^{116}$ M. Caulfield, ${ }^{116}$ S. Chauhan, ${ }^{116}$ M. Chertok, ${ }^{116}$ J. Conway, ${ }^{116}$ R. Conway, ${ }^{116}$ P. T. Cox, ${ }^{116}$ J. Dolen, ${ }^{116}$ R. Erbacher, ${ }^{116}$ M. Gardner, ${ }^{116}$ R. Houtz, ${ }^{116}$ W. Ko, ${ }^{116}$ A. Kopecky, ${ }^{116}$ R. Lander, ${ }^{116}$ O. Mall, ${ }^{116}$ T. Miceli, ${ }^{116}$ R. Nelson, ${ }^{116}$ D. Pellett, ${ }^{116}$ F. Ricci-Tam, ${ }^{116}$ B. Rutherford, ${ }^{116}$ M. Searle, ${ }^{116}$ J. Smith,${ }^{116}$ M. Squires,${ }^{116}$ M. Tripathi, ${ }^{116}$ R. Vasquez Sierra, ${ }^{116}$ R. Yohay,${ }^{116}$ V. Andreev,${ }^{117}$ D. Cline,${ }^{117}$

R. Cousins, ${ }^{117}$ J. Duris, ${ }^{117}$ S. Erhan, ${ }^{117}$ P. Everaerts, ${ }^{117}$ C. Farrell, ${ }^{117}$ J. Hauser, ${ }^{117}$ M. Ignatenko, ${ }^{17}$ C. Jarvis,,${ }^{117}$ G. Rakness, ${ }^{117}$ P. Schlein, ${ }^{117}$,a P. Traczyk, ${ }^{117}$ V. Valuev ${ }^{117}$ M. Weber, ${ }^{117}$ J. Babb,${ }^{118}$ R. Clare, ${ }^{118}$ M. E. Dinardo,${ }^{118}$ J. Ellison, ${ }^{118}$ J. W. Gary, ${ }^{118}$ F. Giordano, ${ }^{118}$ G. Hanson, ${ }^{118}$ H. Liu, ${ }^{118}$ O. R. Long, ${ }^{118}$ A. Luthra, ${ }^{118}$ H. Nguyen, ${ }^{118}$

S. Paramesvaran, ${ }^{118}$ J. Sturdy, ${ }^{118}$ S. Sumowidagdo,${ }^{118}$ R. Wilken, ${ }^{118}$ S. Wimpenny, ${ }^{118}$ W. Andrews,${ }^{119}$

J. G. Branson, ${ }^{119}$ G. B. Cerati, ${ }^{119}$ S. Cittolin, ${ }^{119}$ D. Evans, ${ }^{119}$ A. Holzner, ${ }^{119}$ R. Kelley, ${ }^{119}$ M. Lebourgeois, ${ }^{119}$ J. Letts, ${ }^{119}$ I. Macneill, ${ }^{119}$ B. Mangano, ${ }^{119}$ S. Padhi, ${ }^{119}$ C. Palmer, ${ }^{119}$ G. Petrucciani, ${ }^{119}$ M. Pieri, ${ }^{119}$ M. Sani, ${ }^{119}$

V. Sharma, ${ }^{119}$ S. Simon, ${ }^{119}$ E. Sudano, ${ }^{119}$ M. Tadel, ${ }^{119}$ Y. Tu, ${ }^{119}$ A. Vartak, ${ }^{119}$ S. Wasserbaech,,${ }^{11, \text { bbb }}$

F. Würthwein, ${ }^{119}$ A. Yagil, ${ }^{119}$ J. Yoo, ${ }^{119}$ D. Barge,${ }^{120}$ R. Bellan, ${ }^{120}$ C. Campagnari, ${ }^{120}$ M. D'Alfonso, ${ }^{120}$ T. Danielson, ${ }^{120}$ K. Flowers, ${ }^{120}$ P. Geffert, ${ }^{120}$ C. George, ${ }^{120}$ F. Golf, ${ }^{120}$ J. Incandela, ${ }^{120}$ C. Justus, ${ }^{120}$ P. Kalavase, ${ }^{120}$ D. Kovalskyi, ${ }^{120}$ V. Krutelyov, ${ }^{120}$ S. Lowette, ${ }^{120}$ R. Magaña Villalba, ${ }^{120}$ N. Mccoll, ${ }^{120}$ V. Pavlunin, ${ }^{120}$ J. Ribnik,${ }^{120}$ J. Richman, ${ }^{120}$ R. Rossin, ${ }^{120}$ D. Stuart,${ }^{120}$ W. To, ${ }^{120}$ C. West, ${ }^{120}$ A. Apresyan, ${ }^{121}$ A. Bornheim, ${ }^{121}$ J. Bunn, ${ }^{121}$ Y. Chen, ${ }^{121}$ E. Di Marco, ${ }^{121}$ J. Duarte, ${ }^{121}$ M. Gataullin, ${ }^{121}$ D. Kcira, ${ }^{121}$ Y. Ma, ${ }^{121}$ A. Mott, ${ }^{121}$ H. B. Newman, ${ }^{121}$ C. Rogan, ${ }^{121}$ M. Spiropulu, ${ }^{121}$ V. Timciuc, ${ }^{121}$ J. Veverka, ${ }^{121}$ R. Wilkinson, ${ }^{121}$ S. Xie ${ }^{121}$ Y. Yang, ${ }^{121}$ R. Y. Zhu, ${ }^{121}$ V. Azzolini, ${ }^{122}$ A. Calamba, ${ }^{122}$ R. Carroll, ${ }^{122}$ T. Ferguson, ${ }^{122}$ Y. Iiyama, ${ }^{122}$ D. W. Jang, ${ }^{122}$ Y. F. Liu, ${ }^{122}$ M. Paulini, ${ }^{122}$

H. Vogel, ${ }^{122}$ I. Vorobiev, ${ }^{122}$ J. P. Cumalat,${ }^{123}$ B. R. Drell, ${ }^{123}$ W. T. Ford,${ }^{123}$ A. Gaz, ${ }^{123}$ E. Luiggi Lopez, ${ }^{123}$

J. G. Smith, ${ }^{123}$ K. Stenson, ${ }^{123}$ K. A. Ulmer, ${ }^{123}$ S. R. Wagner, ${ }^{123}$ J. Alexander, ${ }^{124}$ A. Chatterjee, ${ }^{124}$ N. Eggert, ${ }^{124}$

L. K. Gibbons, ${ }^{124}$ B. Heltsley, ${ }^{124}$ W. Hopkins, ${ }^{124}$ A. Khukhunaishvili, ${ }^{124}$ B. Kreis, ${ }^{124}$ N. Mirman, ${ }^{124}$

G. Nicolas Kaufman, ${ }^{124}$ J. R. Patterson, ${ }^{124}$ A. Ryd, ${ }^{124}$ E. Salvati, ${ }^{124}$ W. Sun, ${ }^{124}$ W. D. Teo, ${ }^{124}$ J. Thom, ${ }^{124}$ J. Thompson, ${ }^{124}$ J. Tucker, ${ }^{124}$ Y. Weng, ${ }^{124}$ L. Winstrom, ${ }^{124}$ P. Wittich, ${ }^{124}$ D. Winn, ${ }^{125}$ S. Abdullin, ${ }^{126}$ M. Albrow, ${ }^{126}$ J. Anderson, ${ }^{126}$ L. A. T. Bauerdick, ${ }^{126}$ A. Beretvas, ${ }^{126}$ J. Berryhill, ${ }^{126}$ P. C. Bhat, ${ }^{126}$ K. Burkett, ${ }^{126}$ J. N. Butler, ${ }^{126}$ V. Chetluru, ${ }^{126}$ H. W. K. Cheung, ${ }^{126}$ F. Chlebana, ${ }^{126}$ S. Cihangir, ${ }^{126}$ V. D. Elvira, ${ }^{126}$ I. Fisk, ${ }^{126}$ J. Freeman, ${ }^{126}$ Y. Gao,${ }^{126}$ D. Green, ${ }^{126}$ O. Gutsche, ${ }^{126}$ J. Hanlon, ${ }^{126}$ R. M. Harris, ${ }^{126}$ J. Hirschauer, ${ }^{126}$ B. Hooberman, ${ }^{126}$ S. Jindariani, ${ }^{126}$ M. Johnson, ${ }^{126}$ U. Joshi, ${ }^{126}$ B. Klima, ${ }^{126}$ S. Kunori, ${ }^{126}$ S. Kwan, ${ }^{126}$ C. Leonidopoulos, ${ }^{126, c c c}$ J. Linacre, ${ }^{126}$ D. Lincoln, ${ }^{126}$ R. Lipton, ${ }^{126}$ J. Lykken, ${ }^{126}$ K. Maeshima ${ }^{126}$ J. M. Marraffino, ${ }^{126}$

V. I. Martinez Outschoorn, ${ }^{126}$ S. Maruyama, ${ }^{126}$ D. Mason, ${ }^{126}$ P. McBride, ${ }^{126}$ K. Mishra, ${ }^{126}$ S. Mrenna, ${ }^{126}$ Y. Musienko, ${ }^{126, \text { ddd }}$ C. Newman-Holmes, ${ }^{126}$ V. O’ Dell, ${ }^{126}$ O. Prokofyev, ${ }^{126}$ E. Sexton-Kennedy, ${ }^{126}$ S. Sharma, ${ }^{126}$ W. J. Spalding, ${ }^{126}$ L. Spiegel, ${ }^{126}$ L. Taylor, ${ }^{126}$ S. Tkaczyk, ${ }^{126}$ N. V. Tran, ${ }^{126}$ L. Uplegger, ${ }^{126}$ E. W. Vaandering, ${ }^{126}$

R. Vidal, ${ }^{126}$ J. Whitmore, ${ }^{126} \mathrm{~W}$. Wu, ${ }^{126}$ F. Yang, ${ }^{126}$ J. C. Yun, ${ }^{126}$ D. Acosta, ${ }^{127}$ P. Avery, ${ }^{127}$ D. Bourilkov,${ }^{127}$ M. Chen, ${ }^{127}$ T. Cheng, ${ }^{127}$ S. Das, ${ }^{127}$ M. De Gruttola, ${ }^{127}$ G. P. Di Giovanni, ${ }^{127}$ D. Dobur, ${ }^{127}$ A. Drozdetskiy, ${ }^{127}$ R. D. Field, ${ }^{127}$ M. Fisher, ${ }^{127}$ Y. Fu, ${ }^{127}$ I. K. Furic, ${ }^{127}$ J. Gartner, ${ }^{127}$ J. Hugon, ${ }^{127}$ B. Kim, ${ }^{127}$ J. Konigsberg, ${ }^{127}$ A. Korytov, ${ }^{127}$ A. Kropivnitskaya, ${ }^{127}$ T. Kypreos, ${ }^{127}$ J. F. Low, ${ }^{127}$ K. Matchev, ${ }^{127}$ P. Milenovic,,${ }^{127, \text { eee }}$

G. Mitselmakher, ${ }^{127}$ L. Muniz ${ }^{127}$ M. Park,${ }^{127}$ R. Remington, ${ }^{127}$ A. Rinkevicius, ${ }^{127}$ P. Sellers,${ }^{127}$ N. Skhirtladze,${ }^{127}$ M. Snowball, ${ }^{127}$ J. Yelton, ${ }^{127}$ M. Zakaria, ${ }^{127}$ V. Gaultney, ${ }^{128}$ S. Hewamanage, ${ }^{128}$ L. M. Lebolo, ${ }^{128}$ S. Linn, ${ }^{128}$ P. Markowitz, ${ }^{128}$ G. Martinez, ${ }^{128}$ J. L. Rodriguez, ${ }^{128}$ T. Adams, ${ }^{129}$ A. Askew, ${ }^{129}$ J. Bochenek, ${ }^{129}$ J. Chen, ${ }^{129}$ 
B. Diamond, ${ }^{129}$ S. V. Gleyzer, ${ }^{129}$ J. Haas, ${ }^{129}$ S. Hagopian, ${ }^{129}$ V. Hagopian, ${ }^{129}$ M. Jenkins, ${ }^{129}$ K. F. Johnson, ${ }^{129}$

H. Prosper, ${ }^{129}$ V. Veeraraghavan, ${ }^{129}$ M. Weinberg, ${ }^{129}$ M. M. Baarmand, ${ }^{130}$ B. Dorney, ${ }^{130}$ M. Hohlmann, ${ }^{130}$ H. Kalakhety, ${ }^{130}$ I. Vodopiyanov, ${ }^{130}$ F. Yumiceva, ${ }^{130}$ M. R. Adams, ${ }^{131}$ L. Apanasevich, ${ }^{131}$ Y. Bai, ${ }^{131}$ V.E. Bazterra, ${ }^{131}$ R. R. Betts, ${ }^{131}$ I. Bucinskaite, ${ }^{131}$ J. Callner, ${ }^{131}$ R. Cavanaugh, ${ }^{131}$ O. Evdokimov, ${ }^{131}$ L. Gauthier, ${ }^{131}$ C. E. Gerber, ${ }^{131}$ D. J. Hofman, ${ }^{131}$ S. Khalatyan, ${ }^{131}$ F. Lacroix ${ }^{131}$ C. O'Brien, ${ }^{131}$ C. Silkworth, ${ }^{131}$ D. Strom, ${ }^{131}$ P. Turner,${ }^{131}$ N. Varelas, ${ }^{131}$ U. Akgun, ${ }^{132}$ E. A. Albayrak, ${ }^{132}$ B. Bilki, ${ }^{132, f f f}$ W. Clarida, ${ }^{132}$ F. Duru, ${ }^{132}$ S. Griffiths, ${ }^{132}$ J.-P. Merlo, ${ }^{132}$ H. Mermerkaya, ${ }^{132, \text { ggg }}$ A. Mestvirishvili, ${ }^{132}$ A. Moeller, ${ }^{132}$ J. Nachtman, ${ }^{132}$ C. R. Newsom, ${ }^{132}$ E. Norbeck, ${ }^{132}$ Y. Onel, ${ }^{132}$ F. Ozok, ${ }^{132, x x}$ S. Sen, ${ }^{132}$ P. Tan, ${ }^{132}$ E. Tiras, ${ }^{132}$ J. Wetzel, ${ }^{132}$ T. Yetkin, ${ }^{132}$ K. Yi, ${ }^{132}$ B. A. Barnett, ${ }^{133}$ B. Blumenfeld, ${ }^{133}$ S. Bolognesi, ${ }^{133}$ D. Fehling, ${ }^{133}$ G. Giurgiu, ${ }^{133}$ A. V. Gritsan, ${ }^{133}$ Z. J. Guo, ${ }^{133}$ G. Hu, ${ }^{133}$

P. Maksimovic, ${ }^{133}$ M. Swartz, ${ }^{133}$ A. Whitbeck, ${ }^{133}$ P. Baringer, ${ }^{134}$ A. Bean ${ }^{134}$ G. Benelli, ${ }^{134}$ R. P. Kenny Iii, ${ }^{134}$ M. Murray, ${ }^{134}$ D. Noonan, ${ }^{134}$ S. Sanders, ${ }^{134}$ R. Stringer, ${ }^{134}$ G. Tinti, ${ }^{134}$ J. S. Wood, ${ }^{134}$ A. F. Barfuss, ${ }^{135}$ T. Bolton, ${ }^{135}$ I. Chakaberia, ${ }^{135}$ A. Ivanov, ${ }^{135}$ S. Khalil,${ }^{135}$ M. Makouski, ${ }^{135}$ Y. Maravin, ${ }^{135}$ S. Shrestha, ${ }^{135}$ I. Svintradze, ${ }^{135}$ J. Gronberg, ${ }^{136}$ D. Lange, ${ }^{136}$ F. Rebassoo, ${ }^{136}$ D. Wright, ${ }^{136}$ A. Baden, ${ }^{137}$ B. Calvert, ${ }^{137}$ S. C. Eno, ${ }^{137}$ J. A. Gomez, ${ }^{137}$ N. J. Hadley, ${ }^{137}$ R. G. Kellogg, ${ }^{137}$ M. Kirn, ${ }^{137}$ T. Kolberg,,${ }^{137}$ Y. Lu, ${ }^{137}$ M. Marionneau, ${ }^{137}$ A. C. Mignerey, ${ }^{137}$ K. Pedro, ${ }^{137}$ A. Peterman, ${ }^{137}$ A. Skuja, ${ }^{137}$ J. Temple, ${ }^{137}$ M. B. Tonjes, ${ }^{137}$ S. C. Tonwar, ${ }^{137}$ A. Apyan, ${ }^{138}$ G. Bauer, ${ }^{138}$ J. Bendavid, ${ }^{138}$ W. Busza, ${ }^{138}$ E. Butz,${ }^{138}$ I. A. Cali, ${ }^{138}$ M. Chan, ${ }^{138}$ V. Dutta, ${ }^{138}$ G. Gomez Ceballos, ${ }^{138}$

M. Goncharov, ${ }^{138}$ Y. Kim, ${ }^{138}$ M. Klute, ${ }^{138}$ K. Krajczar, ${ }^{138, \text { hhh }}$ A. Levin, ${ }^{138}$ P. D. Luckey, ${ }^{138}$ T. Ma, ${ }^{138}$ S. Nahn, ${ }^{138}$ C. Paus, ${ }^{138}$ D. Ralph, ${ }^{138}$ C. Roland, ${ }^{138}$ G. Roland,,${ }^{138}$ M. Rudolph, ${ }^{138}$ G. S. F. Stephans, ${ }^{138}$ F. Stöckli, ${ }^{138}$

K. Sumorok, ${ }^{138}$ K. Sung, ${ }^{138}$ D. Velicanu, ${ }^{138}$ E. A. Wenger, ${ }^{138}$ R. Wolf, ${ }^{138}$ B. Wyslouch, ${ }^{138}$ M. Yang, ${ }^{138}$ Y. Yilmaz, ${ }^{138}$ A. S. Yoon, ${ }^{138}$ M. Zanetti, ${ }^{138}$ V. Zhukova, ${ }^{138}$ B. Dahmes, ${ }^{139}$ A. De Benedetti, ${ }^{139}$ G. Franzoni, ${ }^{139}$ A. Gude, ${ }^{139}$ S. C. Kao, ${ }^{139}$ K. Klapoetke, ${ }^{139}$ Y. Kubota, ${ }^{139}$ J. Mans, ${ }^{139}$ N. Pastika, ${ }^{139}$ R. Rusack, ${ }^{139}$ M. Sasseville, ${ }^{139}$ A. Singovsky, ${ }^{139}$ N. Tambe, ${ }^{139}$ J. Turkewitz, ${ }^{139}$ L. M. Cremaldi, ${ }^{140}$ R. Kroeger,${ }^{140}$ L. Perera, ${ }^{140}$ R. Rahmat, ${ }^{140}$ D. A. Sanders, ${ }^{140}$ E. Avdeeva, ${ }^{141}$ K. Bloom, ${ }^{141}$ S. Bose,${ }^{141}$ D. R. Claes, ${ }^{141}$ A. Dominguez, ${ }^{141}$ M. Eads ${ }^{141}$ J. Keller, ${ }^{141}$ I. Kravchenko, ${ }^{141}$ J. Lazo-Flores, ${ }^{141}$ S. Malik, ${ }^{141}$ G. R. Snow, ${ }^{141}$ A. Godshalk, ${ }^{142}$ I. Iashvili, ${ }^{142}$ S. Jain, ${ }^{142}$ A. Kharchilava, ${ }^{142}$ A. Kumar, ${ }^{142}$ S. Rappoccio, ${ }^{142}$ Z. Wan, ${ }^{142}$ G. Alverson, ${ }^{143}$ E. Barberis, ${ }^{143}$ D. Baumgartel,,${ }^{143}$ M. Chasco, ${ }^{143}$ J. Haley, ${ }^{143}$ D. Nash, ${ }^{143}$ T. Orimoto, ${ }^{143}$ D. Trocino, ${ }^{143}$ D. Wood, ${ }^{143}$ J. Zhang, ${ }^{143}$ A. Anastassov, ${ }^{144}$ K. A. Hahn, ${ }^{144}$ A. Kubik, ${ }^{144}$ L. Lusito, ${ }^{144}$ N. Mucia, ${ }^{144}$ N. Odell, ${ }^{144}$ R. A. Ofierzynski, ${ }^{144}$ B. Pollack, ${ }^{144}$ A. Pozdnyakov, ${ }^{144}$ M. Schmitt, ${ }^{144}$ S. Stoynev, ${ }^{144}$ M. Velasco, ${ }^{144}$ S. Won, ${ }^{144}$ D. Berry, ${ }^{145}$ A. Brinkerhoff, ${ }^{145}$ K. M. Chan, ${ }^{145}$ M. Hildreth, ${ }^{145}$ C. Jessop, ${ }^{145}$ D. J. Karmgard, ${ }^{145}$ J. Kolb, ${ }^{145}$ K. Lannon, ${ }^{145}$ W. Luo, ${ }^{145}$ S. Lynch, ${ }^{145}$ N. Marinelli, ${ }^{145}$ D. M. Morse, ${ }^{145}$ T. Pearson, ${ }^{145}$ M. Planer, ${ }^{145}$ R. Ruchti,,${ }^{145}$ J. Slaunwhite, ${ }^{145}$ N. Valls, ${ }^{145}$ M. Wayne, ${ }^{145}$ M. Wolf, ${ }^{145}$ L. Antonelli, ${ }^{146}$ B. Bylsma, ${ }^{146}$ L. S. Durkin, ${ }^{146}$ C. Hill,${ }^{146}$ R. Hughes, ${ }^{146}$ K. Kotov, ${ }^{146}$ T. Y. Ling, ${ }^{146}$ D. Puigh, ${ }^{146}$ M. Rodenburg, ${ }^{146}$ C. Vuosalo, ${ }^{146}$ G. Williams, ${ }^{146}$ B. L. Winer, ${ }^{146}$ E. Berry,${ }^{147}$ P. Elmer, ${ }^{147}$ V. Halyo, ${ }^{147}$ P. Hebda, ${ }^{147}$ J. Hegeman, ${ }^{147}$ A. Hunt, ${ }^{147}$ P. Jindal, ${ }^{147}$ S. A. Koay, ${ }^{147}$ D. Lopes Pegna, ${ }^{147}$ P. Lujan, ${ }^{147}$ D. Marlow, ${ }^{147}$ T. Medvedeva, ${ }_{147}$ M. Mooney, ${ }^{147}$ J. Olsen, ${ }^{147}$ P. Piroué, ${ }^{147}$ X. Quan, ${ }^{147}$ A. Raval, ${ }^{147}$ H. Saka, ${ }^{147}$ D. Stickland, ${ }^{147}$ C. Tully, ${ }^{147}$ J. S. Werner, ${ }^{147}$ S. C. Zenz, ${ }^{147}$ A. Zuranski, ${ }^{147}$ E. Brownson, ${ }^{148}$ A. Lopez, ${ }^{148}$ H. Mendez, ${ }^{148}$ J. E. Ramirez Vargas, ${ }^{148}$ E. Alagoz, ${ }^{149}$ V. E. Barnes, ${ }^{149}$ D. Benedetti, ${ }^{149}$ G. Bolla, ${ }^{149}$ D. Bortoletto, ${ }^{149}$ M. De Mattia, ${ }^{149}$ A. Everett, ${ }^{149}$ Z. Hu ${ }^{149}$ M. Jones, ${ }^{149}$ O. Koybasi, ${ }^{149}$ M. Kress, ${ }^{149}$ A. T. Laasanen,${ }^{149}$

N. Leonardo, ${ }^{149}$ V. Maroussov, ${ }^{149}$ P. Merkel, ${ }^{149}$ D. H. Miller, ${ }^{149}$ N. Neumeister, ${ }^{149}$ I. Shipsey, ${ }^{149}$ D. Silvers, ${ }^{149}$ A. Svyatkovskiy, ${ }^{149}$ M. Vidal Marono, ${ }^{149}$ H. D. Yoo, ${ }^{149}$ J. Zablocki ${ }^{149}$ Y. Zheng, ${ }^{149}$ S. Guragain, ${ }^{150}$ N. Parashar, ${ }^{150}$ A. Adair, ${ }^{151}$ B. Akgun, ${ }^{151}$ C. Boulahouache, ${ }^{151}$ K. M. Ecklund, ${ }^{151}$ F. J. M. Geurts, ${ }^{151}$ W. Li, ${ }^{151}$ B. P. Padley, ${ }^{151}$ R. Redjimi, ${ }^{151}$ J. Roberts, ${ }^{151}$ J. Zabel, ${ }^{151}$ B. Betchart, ${ }^{152}$ A. Bodek, ${ }^{152}$ Y. S. Chung, ${ }^{152}$ R. Covarelli, ${ }^{152}$ P. de Barbaro, ${ }^{152}$ R. Demina, ${ }^{152}$ Y. Eshaq, ${ }^{152}$ T. Ferbel, ${ }^{152}$ A. Garcia-Bellido, ${ }^{152}$ P. Goldenzweig, ${ }^{152}$ J. Han, ${ }^{152}$ A. Harel, ${ }^{152}$ D. C. Miner, ${ }^{152}$ D. Vishnevskiy, ${ }^{152}$ M. Zielinski, ${ }^{152}$ A. Bhatti, ${ }^{153}$ R. Ciesielski, ${ }^{153}$ L. Demortier, ${ }^{153}$ K. Goulianos, ${ }^{153}$ G. Lungu, ${ }^{153}$ S. Malik, ${ }^{153}$ C. Mesropian, ${ }^{153}$ S. Arora, ${ }^{154}$ A. Barker, ${ }^{154}$ J. P. Chou, ${ }^{154}$ C. Contreras-Campana, ${ }^{154}$ E. Contreras-Campana, ${ }^{154}$ D. Duggan, ${ }^{154}$ D. Ferencek, ${ }^{154}$ Y. Gershtein, ${ }^{154}$ R. Gray, ${ }^{154}$ E. Halkiadakis, ${ }^{154}$ D. Hidas, ${ }^{154}$ A. Lath, ${ }^{154}$ S. Panwalkar, ${ }^{154}$ M. Park,${ }^{154}$ R. Patel, ${ }^{154}$ V. Rekovic, ${ }^{154}$ J. Robles,${ }^{154}$ K. Rose, ${ }^{154}$ S. Salur, ${ }^{154}$ S. Schnetzer,${ }^{154}$ C. Seitz, ${ }^{154}$ S. Somalwar, ${ }^{154}$ R. Stone,${ }^{154}$ S. Thomas, ${ }^{154}$ M. Walker,${ }^{154}$ G. Cerizza, ${ }^{155}$ M. Hollingsworth, ${ }^{155}$ S. Spanier, ${ }^{155}$ Z. C. Yang, ${ }^{155}$ A. York, ${ }^{155}$ R. Eusebi, ${ }^{156}$ W. Flanagan, ${ }^{156}$ J. Gilmore, ${ }^{156}$ T. Kamon, ${ }^{156, \text { iii }}$ V. Khotilovich, ${ }^{156}$ R. Montalvo, ${ }^{156}$ I. Osipenkov, ${ }^{156}$ Y. Pakhotin, ${ }^{156}$ A. Perloff, ${ }^{156}$ J. Roe, ${ }^{156}$ A. Safonov, ${ }^{156}$ T. Sakuma, ${ }^{156}$ S. Sengupta,${ }^{156}$ I. Suarez, ${ }^{156}$ A. Tatarinov,${ }^{156}$ D. Toback,${ }^{156}$ N. Akchurin,${ }^{157}$ J. Damgov, ${ }^{157}$ C. Dragoiu, ${ }^{157}$ P. R. Dudero, ${ }^{157}$ C. Jeong, ${ }^{157}$ K. Kovitanggoon, ${ }^{157}$ S. W. Lee, ${ }^{157}$ T. Libeiro, ${ }^{157}$ 
I. Volobouev, ${ }^{157}$ E. Appelt, ${ }^{158}$ A. G. Delannoy, ${ }^{158}$ C. Florez, ${ }^{158}$ S. Greene, ${ }^{158}$ A. Gurrola, ${ }^{158}$ W. Johns, ${ }^{158}$ P. Kurt, ${ }^{158}$ C. Maguire, ${ }^{158}$ A. Melo, ${ }^{158}$ M. Sharma ${ }^{158}$ P. Sheldon, ${ }^{158}$ B. Snook,${ }^{158}$ S. Tuo,${ }^{158}$ J. Velkovska, ${ }^{158}$ M. W. Arenton, ${ }^{159}$ M. Balazs, ${ }^{159}$ S. Boutle ${ }^{159}$ B. Cox,${ }^{159}$ B. Francis, ${ }^{159}$ J. Goodell, ${ }^{159}$ R. Hirosky, ${ }^{159}$ A. Ledovskoy, ${ }^{159}$ C. Lin, ${ }^{159}$ C. Neu, ${ }^{159}$ J. Wood, ${ }^{159}$ S. Gollapinni, ${ }^{160}$ R. Harr, ${ }^{160}$ P. E. Karchin, ${ }^{160}$ C. Kottachchi Kankanamge Don, ${ }^{160}$ P. Lamichhane, ${ }^{160}$ A. Sakharov,${ }^{160}$ M. Anderson, ${ }^{161}$ D. A. Belknap ${ }^{161}$ L. Borrello, ${ }^{161}$ D. Carlsmith, ${ }^{161}$ M. Cepeda, ${ }^{161}$ S. Dasu, ${ }^{161}$ E. Friis, ${ }^{161}$ L. Gray, ${ }^{161}$ K. S. Grogg, ${ }^{161}$ M. Grothe, ${ }^{161}$ R. Hall-Wilton, ${ }^{161}$ M. Herndon, ${ }^{161}$ A. Hervé, ${ }^{161}$ P. Klabbers, ${ }^{161}$ J. Klukas, ${ }^{161}$ A. Lanaro, ${ }^{161}$ C. Lazaridis, ${ }^{161}$ R. Loveless, ${ }^{161}$ A. Mohapatra, ${ }^{161}$ M. U. Mozer,${ }^{161}$ I. Ojalvo, ${ }^{161}$ F. Palmonari, ${ }^{161}$ G. A. Pierro, ${ }^{161}$ I. Ross, ${ }^{161}$ A. Savin, ${ }^{161}$ W. H. Smith, ${ }^{161}$ and J. Swanson ${ }^{161}$

(CMS Collaboration)

\author{
${ }^{1}$ Yerevan Physics Institute, Yerevan, Armenia \\ ${ }^{2}$ Institut für Hochenergiephysik der OeAW, Wien, Austria \\ ${ }^{3}$ National Centre for Particle and High Energy Physics, Minsk, Belarus \\ ${ }^{4}$ Universiteit Antwerpen, Antwerpen, Belgium \\ ${ }^{5}$ Vrije Universiteit Brussel, Brussel, Belgium \\ ${ }^{6}$ Université Libre de Bruxelles, Bruxelles, Belgium \\ ${ }^{7}$ Ghent University, Ghent, Belgium \\ ${ }^{8}$ Université Catholique de Louvain, Louvain-la-Neuve, Belgium \\ ${ }^{9}$ Université de Mons, Mons, Belgium \\ ${ }^{10}$ Centro Brasileiro de Pesquisas Fisicas, Rio de Janeiro, Brazil \\ ${ }^{11}$ Universidade do Estado do Rio de Janeiro, Rio de Janeiro, Brazil \\ ${ }^{12 a}$ Universidade Estadual Paulista, São Paulo, Brazil \\ ${ }^{12 \mathrm{~b}}$ Universidade Federal do ABC, São Paulo, Brazil \\ ${ }^{13}$ Institute for Nuclear Research and Nuclear Energy, Sofia, Bulgaria \\ ${ }^{14}$ University of Sofia, Sofia, Bulgaria \\ ${ }^{15}$ Institute of High Energy Physics, Beijing, China \\ ${ }^{16}$ State Key Laboratory of Nuclear Physics and Technology, Peking University, Beijing, China \\ ${ }^{17}$ Universidad de Los Andes, Bogota, Colombia \\ ${ }^{18}$ Technical University of Split, Split, Croatia \\ ${ }^{19}$ University of Split, Split, Croatia \\ ${ }^{20}$ Institute Rudjer Boskovic, Zagreb, Croatia \\ ${ }^{21}$ University of Cyprus, Nicosia, Cyprus \\ ${ }^{22}$ Charles University, Prague, Czech Republic \\ ${ }^{23}$ Academy of Scientific Research and Technology of the Arab Republic of Egypt, \\ Egyptian Network of High Energy Physics, Cairo, Egypt \\ ${ }^{24}$ National Institute of Chemical Physics and Biophysics, Tallinn, Estonia \\ ${ }^{25}$ Department of Physics, University of Helsinki, Helsinki, Finland \\ ${ }^{26}$ Helsinki Institute of Physics, Helsinki, Finland \\ ${ }^{27}$ Lappeenranta University of Technology, Lappeenranta, Finland \\ ${ }^{28}$ DSM/IRFU, CEA/Saclay, Gif-sur-Yvette, France \\ ${ }^{29}$ Laboratoire Leprince-Ringuet, Ecole Polytechnique, IN2P3-CNRS, Palaiseau, France \\ ${ }^{30}$ Institut Pluridisciplinaire Hubert Curien, Université de Strasbourg, Université de Haute Alsace Mulhouse, \\ CNRS/IN2P3, Strasbourg, France \\ ${ }^{31}$ Université de Lyon, Université Claude Bernard Lyon 1, CNRS-IN2P3, Institut de Physique Nucléaire de Lyon, \\ Villeurbanne, France \\ ${ }^{32}$ Institute of High Energy Physics and Informatization, Tbilisi State University, Tbilisi, Georgia \\ ${ }^{33}$ RWTH Aachen University, I. Physikalisches Institut, Aachen, Germany \\ ${ }^{34}$ RWTH Aachen University, III. Physikalisches Institut A, Aachen, Germany \\ ${ }^{35}$ RWTH Aachen University, III. Physikalisches Institut B, Aachen, Germany \\ ${ }^{36}$ Deutsches Elektronen-Synchrotron, Hamburg, Germany \\ ${ }^{37}$ University of Hamburg, Hamburg, Germany \\ ${ }^{38}$ Institut für Experimentelle Kernphysik, Karlsruhe, Germany \\ ${ }^{39}$ Institute of Nuclear Physics "Demokritos", Aghia Paraskevi, Greece \\ ${ }^{40}$ University of Athens, Athens, Greece \\ ${ }^{41}$ University of Ioánnina, Ioánnina, Greece \\ ${ }^{42}$ KFKI Research Institute for Particle and Nuclear Physics, Budapest, Hungary \\ ${ }^{43}$ Institute of Nuclear Research ATOMKI, Debrecen, Hungary \\ ${ }^{44}$ University of Debrecen, Debrecen, Hungary
}


${ }^{45}$ Panjab University, Chandigarh, India

${ }^{46}$ University of Delhi, Delhi, India

${ }^{47}$ Saha Institute of Nuclear Physics, Kolkata, India

${ }^{48}$ Bhabha Atomic Research Centre, Mumbai, India

${ }^{49}$ Tata Institute of Fundamental Research-EHEP, Mumbai, India

${ }^{50}$ Tata Institute of Fundamental Research-HECR, Mumbai, India

${ }^{51}$ Institute for Research in Fundamental Sciences (IPM), Tehran, Iran

${ }^{52 a}$ INFN Sezione di Bari, Bari, Italy

${ }^{52 \mathrm{~b}}$ Università di Bari, Bari, Italy

${ }^{52 \mathrm{c}}$ Politecnico di Bari, Bari, Italy

${ }^{53}$ INFN Sezione di Bologna, Bologna, Italy

${ }^{53 \mathrm{~b}}$ Università di Bologna, Bologna, Italy

${ }^{54 a}$ INFN Sezione di Catania, Catania, Italy

${ }^{54 \mathrm{~b}}$ Università di Catania, Catania, Italy

${ }^{55}$ a INFN Sezione di Firenze, Firenze, Italy

${ }^{55 \mathrm{~b}}$ Università di Firenze, Firenze, Italy

${ }^{56}$ INFN Laboratori Nazionali di Frascati, Frascati, Italy

${ }^{57 a}$ INFN Sezione di Genova, Genova, Italy

${ }^{57 b}$ Università di Genova, Genova, Italy

${ }^{58 a}$ INFN Sezione di Milano-Bicocca, Milano, Italy

${ }^{58 \mathrm{~b}}$ Università di Milano-Bicocca, Milano, Italy

${ }^{59 a}$ INFN Sezione di Napoli, Napoli, Italy

${ }^{59 b}$ Università di Napoli 'Federico II', Napoli, Italy

${ }^{59 \mathrm{c}}$ Università della Basilicata (Potenza), Napoli, Italy

${ }^{59 d}$ Università G. Marconi (Roma), Napoli, Italy

${ }^{60 a}$ INFN Sezione di Padova, Padova, Italy

${ }^{60 \mathrm{~b}}$ Università di Padova, Padova, Italy

${ }^{60 c}$ Università di Trento (Trento), Padova, Italy

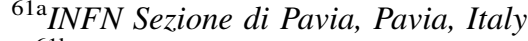

${ }^{61 \mathrm{~b}}$ Università di Pavia, Pavia, Italy

${ }^{62 a}$ INFN Sezione di Perugia, Perugia, Italy

${ }^{62 \mathrm{~b}}$ Università di Perugia, Perugia, Italy

${ }^{63 a}$ INFN Sezione di Pisa, Pisa, Italy

${ }^{63 \mathrm{~b}}$ Università di Pisa, Pisa, Italy

${ }^{63 \mathrm{c}}$ Scuola Normale Superiore di Pisa, Pisa, Italy

${ }^{64 a}$ INFN Sezione di Roma, Roma, Italy

${ }^{64 \mathrm{~b}}$ Università di Roma, Roma, Italy

${ }^{65}$ a INFN Sezione di Torino, Torino, Italy

${ }^{65 \mathrm{~b}}$ Università di Torino, Torino, Italy

${ }^{65 c}$ Università del Piemonte Orientale (Novara), Torino, Italy

${ }^{66 a}$ INFN Sezione di Trieste, Trieste, Italy

${ }^{66 \mathrm{~b}}$ Università di Trieste, Trieste, Italy

${ }^{67}$ Kangwon National University, Chunchon, Korea

${ }^{68}$ Kyungpook National University, Daegu, Korea

${ }^{69}$ Chonnam National University, Institute for Universe and Elementary Particles, Kwangju, Korea

${ }^{70}$ Korea University, Seoul, Korea

${ }^{71}$ University of Seoul, Seoul, Korea

${ }^{72}$ Sungkyunkwan University, Suwon, Korea

${ }^{73}$ Vilnius University, Vilnius, Lithuania

${ }^{74}$ Centro de Investigacion y de Estudios Avanzados del IPN, Mexico City, Mexico

${ }^{75}$ Universidad Iberoamericana, Mexico City, Mexico

${ }^{76}$ Benemerita Universidad Autonoma de Puebla, Puebla, Mexico

${ }^{77}$ Universidad Autónoma de San Luis Potosí, San Luis Potosí, Mexico

${ }^{78}$ University of Auckland, Auckland, New Zealand

${ }^{79}$ University of Canterbury, Christchurch, New Zealand

${ }^{80}$ National Centre for Physics, Quaid-I-Azam University, Islamabad, Pakistan

${ }^{81}$ National Centre for Nuclear Research, Swierk, Poland

${ }^{82}$ Institute of Experimental Physics, Faculty of Physics, University of Warsaw, Warsaw, Poland

${ }^{83}$ Laboratório de Instrumentação e Física Experimental de Partículas, Lisboa, Portugal

${ }^{84}$ Joint Institute for Nuclear Research, Dubna, Russia

${ }^{85}$ Petersburg Nuclear Physics Institute, Gatchina (St. Petersburg), Russia 
${ }^{86}$ Institute for Nuclear Research, Moscow, Russia

${ }^{87}$ Institute for Theoretical and Experimental Physics, Moscow, Russia

${ }^{88}$ P.N. Lebedev Physical Institute, Moscow, Russia

${ }^{89}$ Skobeltsyn Institute of Nuclear Physics, Lomonosov Moscow State University, Moscow, Russia

${ }^{90}$ State Research Center of Russian Federation, Institute for High Energy Physics, Protvino, Russia

${ }^{91}$ University of Belgrade, Faculty of Physics and Vinca Institute of Nuclear Sciences, Belgrade, Serbia

${ }^{92}$ Centro de Investigaciones Energéticas Medioambientales y Tecnológicas (CIEMAT), Madrid, Spain

${ }^{93}$ Universidad Autónoma de Madrid, Madrid, Spain

${ }^{94}$ Universidad de Oviedo, Oviedo, Spain

${ }^{95}$ Instituto de Física de Cantabria (IFCA), CSIC-Universidad de Cantabria, Santander, Spain

${ }^{96}$ CERN, European Organization for Nuclear Research, Geneva, Switzerland

${ }^{97}$ Paul Scherrer Institut, Villigen, Switzerland

${ }^{98}$ Institute for Particle Physics, ETH Zurich, Zurich, Switzerland

${ }^{99}$ Universität Zürich, Zurich, Switzerland

${ }^{100}$ National Central University, Chung-Li, Taiwan

${ }^{101}$ National Taiwan University (NTU), Taipei, Taiwan

${ }^{102}$ Chulalongkorn University, Bangkok, Thailand

${ }^{103}$ Cukurova University, Adana, Turkey

${ }^{104}$ Middle East Technical University, Physics Department, Ankara, Turkey

${ }^{105}$ Bogazici University, Istanbul, Turkey

${ }^{106}$ Istanbul Technical University, Istanbul, Turkey

${ }^{107}$ National Scientific Center, Kharkov Institute of Physics and Technology, Kharkov, Ukraine

${ }^{108}$ University of Bristol, Bristol, United Kingdom

${ }^{109}$ Rutherford Appleton Laboratory, Didcot, United Kingdom

${ }^{110}$ Imperial College, London, United Kingdom

${ }^{111}$ Brunel University, Uxbridge, United Kingdom

${ }^{112}$ Baylor University, Waco, Texas, USA

${ }^{113}$ The University of Alabama, Tuscaloosa, Alabama, USA

${ }^{114}$ Boston University, Boston, Massachusetts, USA

${ }^{115}$ Brown University, Providence, New Jersey, USA

${ }^{116}$ University of California, Davis, Davis, California, USA

${ }^{117}$ University of California, Los Angeles, Los Angeles, California, USA

${ }^{118}$ University of California, Riverside, Riverside, California, USA

${ }^{119}$ University of California, San Diego, La Jolla, California, USA

${ }^{120}$ University of California, Santa Barbara, Santa Barbara, California, USA

${ }^{121}$ California Institute of Technology, Pasadena, California, USA

${ }^{122}$ Carnegie Mellon University, Pittsburgh, Pennsylvania, USA

${ }^{123}$ University of Colorado at Boulder, Boulder, Colorado, USA

${ }^{124}$ Cornell University, Ithaca, New York, USA

${ }^{125}$ Fairfield University, Fairfield, Connecticut, USA

${ }^{126}$ Fermi National Accelerator Laboratory, Batavia, Illinois, USA

${ }^{127}$ University of Florida, Gainesville, Florida, USA

${ }^{128}$ Florida International University, Miami, Florida, USA

${ }^{129}$ Florida State University, Tallahassee, Florida, USA

${ }^{130}$ Florida Institute of Technology, Melbourne, Florida, USA

${ }^{131}$ University of Illinois at Chicago (UIC), Chicago, Illinois, USA

${ }^{132}$ The University of Iowa, Iowa City, Iowa, USA

${ }^{133}$ Johns Hopkins University, Baltimore, Maryland, USA

${ }^{134}$ The University of Kansas, Lawrence, Kansas, USA

${ }^{135}$ Kansas State University, Manhattan, Kansas, USA

${ }^{136}$ Lawrence Livermore National Laboratory, Livermore, California, USA

${ }^{137}$ University of Maryland, College Park, Maryland, USA

${ }^{138}$ Massachusetts Institute of Technology, Cambridge, Massachusetts, USA

${ }^{139}$ University of Minnesota, Minneapolis, Minnesota, USA

${ }^{140}$ University of Mississippi, Oxford, Mississippi, USA

${ }^{141}$ University of Nebraska-Lincoln, Lincoln, Nebraska, USA

${ }^{142}$ State University of New York at Buffalo, Buffalo, New York, USA

${ }^{143}$ Northeastern University, Boston, Massachusetts, USA

${ }^{144}$ Northwestern University, Evanston, Illinois, USA

${ }^{145}$ University of Notre Dame, Notre Dame, Indiana, USA

${ }^{146}$ The Ohio State University, Columbus, Ohio, USA 


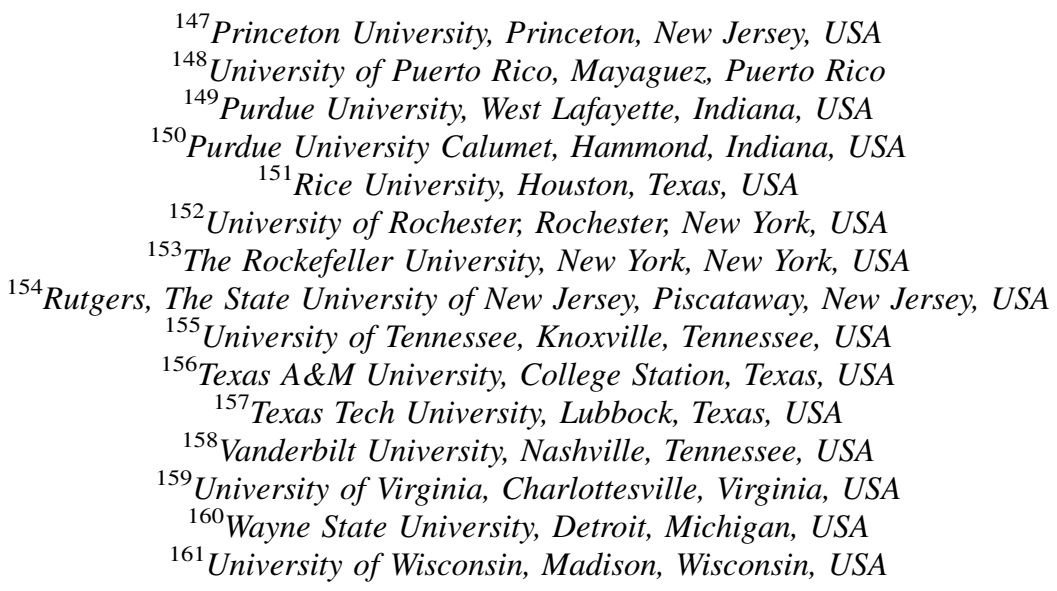

${ }^{\mathrm{a}}$ Deceased.

${ }^{\mathrm{b}}$ Also at Vienna University of Technology, Vienna, Austria.

${ }^{\mathrm{c}}$ Also at CERN, European Organization for Nuclear Research, Geneva, Switzerland.

${ }^{\mathrm{d} A l s o}$ at National Institute of Chemical Physics and Biophysics, Tallinn, Estonia.

${ }^{\mathrm{e}}$ Also at California Institute of Technology, Pasadena, CA, USA.

${ }^{\mathrm{f}}$ Also at Laboratoire Leprince-Ringuet, Ecole Polytechnique, IN2P3-CNRS, Palaiseau, France.

${ }^{\mathrm{g}}$ Also at Suez Canal University, Suez, Egypt.

${ }^{\mathrm{h}}$ Also at Zewail City of Science and Technology, Zewail, Egypt.

${ }^{\mathrm{i}}$ Also at Cairo University, Cairo, Egypt.

${ }^{\mathrm{j} A l s o}$ at Fayoum University, El-Fayoum, Egypt.

${ }^{\mathrm{k}}$ Also at British University in Egypt, Cairo, Egypt.

${ }^{1}$ Now at Ain Shams University, Cairo, Egypt.

${ }^{\mathrm{m}}$ Also at National Centre for Nuclear Research, Swierk, Poland.

${ }^{\mathrm{n}}$ Also at Université de Haute Alsace, Mulhouse, France.

${ }^{\circ}$ Also at Joint Institute for Nuclear Research, Dubna, Russia.

${ }^{\mathrm{p}}$ Also at Skobeltsyn Institute of Nuclear Physics, Lomonosov Moscow State University, Moscow, Russia.

${ }^{\mathrm{q}}$ Also at Brandenburg University of Technology, Cottbus, Germany.

${ }^{\mathrm{r}}$ Also at The University of Kansas, Lawrence, KS, USA.

${ }^{\mathrm{s}}$ Also at Institute of Nuclear Research ATOMKI, Debrecen, Hungary.

${ }^{t}$ Also at Eötvös Loránd University, Budapest, Hungary.

${ }^{\mathrm{u}}$ Also at Tata Institute of Fundamental Research - HECR, Mumbai, India.

${ }^{\mathrm{v}}$ Now at King Abdulaziz University, Jeddah, Saudi Arabia.

${ }^{\mathrm{w}}$ Also at University of Visva-Bharati, Santiniketan, India.

${ }^{\mathrm{x}}$ Also at Sharif University of Technology, Tehran, Iran.

${ }^{\mathrm{y}}$ Also at Isfahan University of Technology, Isfahan, Iran.

${ }^{\mathrm{z}}$ Also at Shiraz University, Shiraz, Iran.

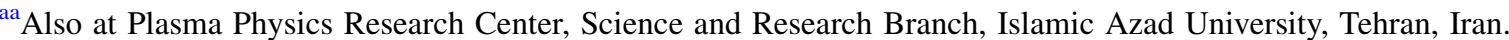



${ }^{\mathrm{cc}}$ Also at Università degli Studi Guglielmo Marconi, Roma, Italy.

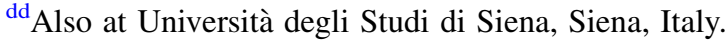

${ }^{e e}$ Also at University of Bucharest, Faculty of Physics, Bucuresti-Magurele, Romania.

${ }^{\mathrm{ff}}$ Also at Faculty of Physics, University of Belgrade, Belgrade, Serbia.

${ }^{\mathrm{gg}}$ Also at University of California, Los Angeles, CA, USA.

${ }^{\text {hh }}$ Also at Scuola Normale e Sezione dell'INFN, Pisa, Italy.

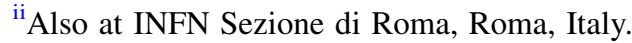

${ }^{\mathrm{jj}}$ Also at University of Athens, Athens, Greece.

${ }^{\mathrm{kk}}$ Also at Rutherford Appleton Laboratory, Didcot, United Kingdom.

${ }^{11}$ Also at Paul Scherrer Institut, Villigen, Switzerland.

${ }^{\mathrm{mm}}$ Also at Institute for Theoretical and Experimental Physics, Moscow, Russia.

${ }^{n n}$ Also at Albert Einstein Center for Fundamental Physics, Bern, Switzerland.




${ }^{\mathrm{pp} A l s o}$ at Adiyaman University, Adiyaman, Turkey.

${ }^{\mathrm{qq}}$ Also at Izmir Institute of Technology, Izmir, Turkey.

${ }^{\mathrm{rr}}$ Also at The University of Iowa, Iowa City, IA, USA.

${ }^{\text {ss } A l s o ~ a t ~ M e r s i n ~ U n i v e r s i t y, ~ M e r s i n, ~ T u r k e y . ~}$

${ }^{t t}$ Also at Ozyegin University, Istanbul, Turkey.

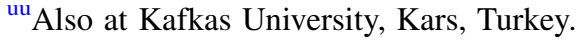

${ }^{\mathrm{vv}}$ Also at Suleyman Demirel University, Isparta, Turkey.

${ }^{\text {ww }}$ Also at Ege University, Izmir, Turkey.

${ }^{\mathrm{xx}}$ Also at Mimar Sinan University, Istanbul, Istanbul, Turkey.



${ }^{\mathrm{zz}}$ Also at School of Physics and Astronomy, University of Southampton, Southampton, United Kingdom.

${ }^{\text {aaa }}$ Also at INFN Sezione di Perugia, Università di Perugia, Perugia, Italy.

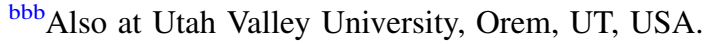

${ }^{\mathrm{ccc}}$ Now at University of Edinburgh, Scotland, Edinburgh, United Kingdom.

${ }^{\text {ddd }}$ Also at Institute for Nuclear Research, Moscow, Russia.

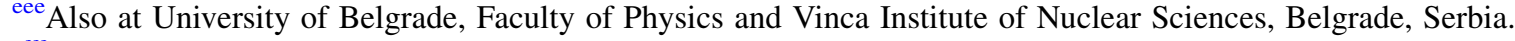

${ }^{\mathrm{fff}}$ Also at Argonne National Laboratory, Argonne, IL, USA.

${ }^{\text {ggg }}$ Also at Erzincan University, Erzincan, Turkey.

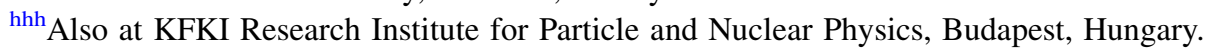

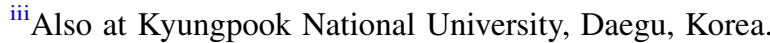

